\title{
The headlight cloud in NGC 628: An extreme giant molecular cloud in a typical galaxy disk ${ }^{\star}$
}

Cinthya N. Herrera ${ }^{1}$, Jérôme Pety ${ }^{1,2}$, Annie Hughes ${ }^{3,4}$, Sharon E. Meidt ${ }^{5,6}$, Kathryn Kreckel $^{5}$, Miguel Querejeta ${ }^{7,8}$, Toshiki Saito ${ }^{5}$, Philipp Lang ${ }^{5}$, María Jesús Jiménez-Donaire ${ }^{9}$, Ismael Pessa ${ }^{5}$, Diane Cormier ${ }^{10}$, Antonio Usero ${ }^{9}$, Kazimierz Sliwa ${ }^{5}$, Christopher Faesi ${ }^{5}$, Guillermo A. Blanc ${ }^{11,12}$, Frank Bigiel ${ }^{13}$, Mélanie Chevance ${ }^{14}$,

Daniel A. Dale ${ }^{15}$, Kathryn Grasha ${ }^{16}$, Simon C. O. Glover ${ }^{17}$, Alexander P. S. Hygate ${ }^{5,14}$, J. M. Diederik Kruijssen ${ }^{14}$, Adam K. Leroy ${ }^{18}$, Erik Rosolowsky ${ }^{19}$, Eva Schinnerer ${ }^{5}$, Andreas Schruba ${ }^{20}$, Jiayi Sun ${ }^{18}$, and Dyas Utomo ${ }^{18}$

${ }^{1}$ Institut de Radioastronomie Millimétrique, 300 rue de la Piscine, 38406 Saint-Martin-d'Hères Cedex, France e-mail: herrera@iram.fr

2 Sorbonne Université, Observatoire de Paris, Université PSL, CNRS, LERMA, 75014 Paris, France

3 CNRS, IRAP, 9 Av. du Colonel Roche, BP 44346, 31028 Toulouse Cedex 4, France

${ }^{4}$ Université de Toulouse, UPS-OMP, IRAP, 31028 Toulouse Cedex 4, France

5 Max Planck Institut für Astronomie, Königstuhl 17, 69117 Heidelberg, Germany

6 Sterrenkundig Observatorium, Universiteit Gent, Krijgslaan 281 S9, 9000 Gent, Belgium

7 European Southern Observatory, Karl-Schwarzschild-Str. 2, 85748 Garching, Germany

8 Observatorio Astronómico Nacional (IGN), C/ Alfonso XII 3, 28014 Madrid, Spain

9 Harvard \& Smithsonian | Center for Astrophysics, 60 Garden St. MS-78, 02138 Cambridge, USA

10 The Observatories of the Carnegie Institution for Science, 813 Santa Barbara St., Pasadena, CA 91101, USA

11 Departamento de Astronomía, Universidad de Chile, Camino del Observatorio 1515, Las Condes, Santiago, Chile

12 AIM, CEA, CNRS, Université Paris-Saclay, Université Paris Diderot, Sorbonne Paris Cité, 91191 Gif-sur-Yvette, France

13 Argelander-Institut für Astronomie, Universität Bonn, Auf dem Hügel 71, 53121 Bonn, Germany

14 Astronomisches Rechen-Institut, Zentrum für Astronomie der Universität Heidelberg, Mönchhofstraße 12-14, 69120 Heidelberg, Germany

15 Department of Physics \& Astronomy, University of Wyoming, Laramie, WY 82071, USA

16 Research School of Astronomy and Astrophysics, Australian National University, Canberra, ACT 2601, Australia

17 Institut für Theoretische Astrophysik, Zentrum für Astronomie der Universität Heidelberg, Albert-Ueberle-Strasse 2, 69120 Heidelberg, Germany

18 Department of Astronomy, The Ohio State University, 140 West 18th Ave, Columbus, OH 43210, USA

19 Department of Physics, University of Alberta, Edmonton, AB T6G 2E1, Canada

20 Max-Planck-Institut für Extraterrestrische Physik, Giessenbachstraße 1, 85748 Garching, Germany

Received 10 June 2019 / Accepted 28 October 2019

\section{ABSTRACT}

Context. Cloud-scale surveys of molecular gas reveal the link between giant molecular cloud properties and star formation across a range of galactic environments. Cloud populations in galaxy disks are considered to be representative of the normal star formation process, while galaxy centers tend to harbor denser gas that exhibits more extreme star formation. At high resolution, however, molecular clouds with exceptional gas properties and star formation activity may also be observed in normal disk environments. In this paper we study the brightest cloud traced in $\mathrm{CO}(2-1)$ emission in the disk of nearby spiral galaxy NGC 628 .

Aims. We characterize the properties of the molecular and ionized gas that is spatially coincident with an extremely bright $\mathrm{H}$ II region in the context of the NGC 628 galactic environment. We investigate how feedback and large-scale processes influence the properties of the molecular gas in this region.

Methods. High-resolution ALMA observations of $\mathrm{CO}(2-1)$ and $\mathrm{CO}(1-0)$ emission were used to characterize the mass and dynamical state of the "headlight" molecular cloud. The characteristics of this cloud are compared to the typical properties of molecular clouds in NGC 628. A simple large velocity gradient (LVG) analysis incorporating additional ALMA observations of ${ }^{13} \mathrm{CO}(1-0), \mathrm{HCO}^{+}(1-0)$, and $\mathrm{HCN}(1-0)$ emission was used to constrain the beam-diluted density and temperature of the molecular gas. We analyzed the MUSE spectrum using Starburst99 to characterize the young stellar population associated with the H II region.

Results. The unusually bright headlight cloud is massive $\left(1-2 \times 10^{7} M_{\odot}\right)$, with a beam-diluted density of $n_{\mathrm{H}_{2}}=5 \times 10^{4} \mathrm{~cm}^{-3}$ based on LVG modeling. It has a low virial parameter, suggesting that the $\mathrm{CO}$ emission associated with this cloud may be overluminous due to heating by the $\mathrm{H}$ II region. A young (2-4 Myr) stellar population with mass $3 \times 10^{5} M_{\odot}$ is associated.

Conclusions. We argue that the headlight cloud is currently being destroyed by feedback from young massive stars. Due to the large mass of the cloud, this phase of the its evolution is long enough for the impact of feedback on the excitation of the gas to be observed. The high mass of the headlight cloud may be related to its location at a spiral co-rotation radius, where gas experiences reduced galactic shear compared to other regions of the disk and receives a sustained inflow of gas that can promote the mass growth of the cloud.

Key words. ISM: clouds - HII regions - stars: formation - Galaxy: disk - Galaxy: kinematics and dynamics

\footnotetext{
* The reduced data cubes are only available at https://doi.org/10.11570/20.0001, in the page https://www. canfar. net/storage/list/AstroDataCitationDOI/CISTI.CANFAR/20.0001/data
} 


\section{Introduction}

NGC 628 (M 74) is a nearby ( $d=9.6$ Mpc, Kreckel et al. 2017), almost face-on $\left(i \sim 9^{\circ}\right.$, Blanc et al. 2013), SAc-type granddesign spiral galaxy with a stellar mass of $M_{\star}=1.5-2 \times 10^{10} M_{\odot}$ (Querejeta et al. 2015; Leroy et al. 2019) and a moderate global star formation rate (SFR) of $\sim 2 M_{\odot} \mathrm{yr}^{-1}$ (Sánchez et al. 2011). A well-known Messier object, NGC 628 is one of about ten galaxies that has been observed by nearly all recent major surveys of the interstellar gas and dust in nearby galaxies, including THINGS, HERACLES, SINGS, KINGFISH, and EMPIRE (Walter et al. 2008; Leroy et al. 2009; Kennicutt et al. 2003, 2011; Bigiel et al. 2016). NGC 628 is also a popular target for observational studies of ionized gas and star formation in the local Universe, with existing wide-field WFPC3/ACS observations by the Hubble Space Telescope (HST) Legacy Extragalactic UV Survey (LEGUS) program (Calzetti et al. 2015) that enable a detailed characterization of the population of stellar clusters and associations in NGC 628, as well as highresolution, wide-field optical Integral Field Unit (IFU) imaging by the VENGA survey and CFHT/SITELLE (Blanc et al. 2013; Rousseau-Nepton et al. 2018).

Using the Multi Unit Spectroscopic Explorer (MUSE) optical IFU instrument on the Very Large Telescope (VLT), Kreckel et al. $(2016,2018)$ recently noted an extremely bright $\mathrm{H}$ II region in the outer part of the galaxy (also visible but uncommented on in earlier H $\alpha$ mapping; e.g., Ferguson et al. 1998; Lelièvre \& Roy 2000). The source stands out as a bright compact peak in the MUSE H $\alpha$ map. It is two orders of magnitude brighter than the mean H II region in the galaxy population and twice as bright as the next most luminous source identified by Kreckel et al. (2018, see their Fig. 1). The H II region has an equivalent radius of $142 \mathrm{pc}$, a velocity dispersion of $50 \mathrm{~km} \mathrm{~s}^{-1}$, and an $\mathrm{H} \alpha$ luminosity of $6.3 \times 10^{39} \mathrm{erg} \mathrm{s}^{-1}$, which corresponds to a local SFR of $\sim 0.034 M_{\odot} \mathrm{yr}^{-1}$, adopting the calibration of Kennicutt \& Evans (2012). This bright H II region is associated with bright compact infrared emission in Spitzer and Herschel maps (see, e.g., Aniano et al. 2012). It appears as the brightest spot in the galaxy in maps of WISE $12 \mu \mathrm{m}$ and $22 \mu \mathrm{m}$ emission (Leroy et al. 2019). The region thus exhibits the classic observational signatures of a large population of luminous young stars that are still associated with a large reservoir of interstellar gas and dust.

The association with gas is borne out by our new Atacama Large Millime- ter/submillimeter Array (ALMA) CO observations. We observed NGC 628 at $1^{\prime \prime} \sim 50$ pc resolution in $\mathrm{CO}(2-1)$ as part of a PHANGS-ALMA survey ${ }^{1}$ (PI: E. Schinnerer; co-PIs: A. Hughes, A. K. Leroy, A. Schruba, E. Rosolowsky). These NGC 628 CO maps have already appeared in Leroy et al. (2016), Sun et al. (2018), Kreckel et al. (2018), and Utomo et al. (2018). The ALMA data reveal an exceptionally bright CO peak spatially coincident with the $\mathrm{H}$ II region (the association is particularly striking in Fig. 1 of Kreckel et al. 2018). While the source was visible in earlier CO maps (e.g., Wakker \& Adler 1995; Helfer et al. 2003; Leroy et al. 2009; Rebolledo et al. 2015), ALMA shows it to be stunningly compact and bright. This source is thus a compact dust-enshrouded collection of many massive young stars still associated with what appears to be the most massive molecular cloud in NGC 628. This object is even more remarkable because of its location at large galactocentric radius,

\footnotetext{
1 Physics at High Angular resolution in Nearby GalaxieS (PHANGS) is an international collaboration whose aim is to understand the interplay of the small-scale physics of gas and star formation with galactic structure and evolution: http://www . phangs.org
}

which makes it distinct from the gas-rich intensely star-forming regions that are commonly identified in galaxy centers.

A preliminary census of the disk environments in the PHANGS-ALMA galaxy sample suggests that this type of object is relatively rare, but not unique. To understand the internal and external factors that can influence the formation and evolution of such massive molecular clouds and their extraordinary H II regions away from galaxy centers, a thorough examination of local physical conditions in the star-forming gas is essential. In this paper, we present the first such study, focusing on the CO-bright cloud in NGC 628 as a prototype. We named this object the headlight cloud because it appears as a bright spot in an otherwise almost dark (unsaturated) map of the $\mathrm{H} \alpha$ and $\mathrm{CO}$ emission.

Several factors make the headlight cloud the ideal candidate for this preliminary study. As noted above, NGC 628 is one of a limited (but growing) set of targets with information for a suite of millimeter and optical emission lines that can be used to constrain the physical properties of the molecular gas and the young stellar population. Relevant global galaxy properties (e.g., SFR) and the galaxy-scale gas dynamics in NGC 628 can be precisely characterized using the wealth of multi-wavelength imaging data. NGC 628 is also part of LEGUS (Calzetti et al. 2015). Studies using the LEGUS cluster catalog present a detailed quantitative overview of the population of NGC 628, and the ability of the NGC 628 gas disk to form clusters and regulate their evolution (Adamo et al. 2017; Grasha et al. 2015, 2017; Ryon et al. 2017). Nevertheless, the region hosting the headlight cloud lies just outside the coverage of the final multi-wavelength LEGUS mosaic.

Our goal in this paper is to measure the physical conditions in the molecular gas of the headlight cloud and to quantitatively describe the associated star formation activity. By comparing the measurements for the headlight cloud to the rest of the galaxy, we aim to build a picture of what may have prompted the growth of such a massive cloud and its extreme star formation event. The paper is structured as follows. In Sect. 2 we present the observational data that we use. In Sect. 3 we describe the properties of the molecular gas. In Sect. 4 we discuss the stellar formation and feedback in the headlight cloud based on the MUSE data. In Sect. 5 we investigate the galactic environment of the headlight cloud. In Sect. 6 we discuss the results. Our conclusions are summarized in Sect. 7.

\section{Observations}

NGC 628 was observed with ALMA in Chile during ALMA's Cycle 1 (ID: 2012.1.00650.S, PI: E. Schinnerer) and Cycle 2 (ID: 2013.1.00532.S, PI: E. Schinnerer). The MUSE and CO(2-1) data have previously appeared elsewhere (see Sect. 1); however, the $3 \mathrm{~mm}$ line observations are presented here for the first time.

\section{1. $1 \mathrm{~mm}$ lines}

The observational strategy, calibration, and imaging of the interferometric data, array combination, and data product delivery of the PHANGS ALMA CO(2-1) data are described in a forthcoming dedicated PHANGS-ALMA survey paper (Leroy et al., in prep.). In Sects. 2.1.1 and 2.1.2 we briefly summarize the key steps for completeness. The present paper is the appropriate citation for the reduction of the PHANGS-ALMA total power data, which we present in detail in Appendix A and summarize below. The strategy that we used to reduce the $\mathrm{CO}(2-1)$ total power data 
for NGC 628 has been adopted as the basis for the PHANGSALMA total power processing pipeline.

\subsubsection{Observations}

ALMA Band 6 observations were obtained during Cycle 1 to image the emission of ${ }^{12} \mathrm{CO}(2-1)$ (hereafter $\left.\mathrm{CO}(2-1)\right)$, $\mathrm{CS}(5-4)$, and the $1 \mathrm{~mm}$ continuum. The frequency ranges covered in the source frame were $229.6-230.5 \mathrm{GHz}$ and $231.0-232.8 \mathrm{GHz}$ in the lower sideband (LSB), and $244.0-245.9 \mathrm{GHz}$ and $246.7-246.8 \mathrm{GHz}$ in the upper sideband (USB). The targeted field of view was a rectangular area of $240^{\prime \prime} \times 175^{\prime \prime}$ centered on the galaxy nucleus.

In order to recover all the spatial scales of the $\mathrm{CO}(2-1)$ emission, $12 \mathrm{~m}, 7 \mathrm{~m}$, and total power observations were performed. On-the-fly observations with three single-dish $12 \mathrm{~m}$ antennas delivered the total power. The off-source position was chosen at the offset $\left[240^{\prime \prime}, 420^{\prime \prime}\right]$ from the phase center located at $\alpha=$ $01^{\mathrm{h}} 36^{\mathrm{m}} 41 \mathrm{~s} 72, \delta=+15^{\circ} 46^{\prime} 59^{\prime \prime} \cdot 3$ in the equatorial $\mathrm{J} 2000$ frame. Mosaics with a total of 149 and 95 pointings were observed with 27 to 38 antennas of the main $12 \mathrm{~m}$ array and with 7 to 9 antennas of the $7 \mathrm{~m}$ array, respectively. A total of 8,10 , and 24 execution blocks were observed for the $12 \mathrm{~m}, 7 \mathrm{~m}$, and total power arrays, respectively.

\subsubsection{Reduction and imaging}

The $\mathrm{CO}(2-1)$ observations were part of the pilot program for PHANGS-ALMA. Leroy et al. (in prep.) describe the selection, reduction, and imaging of the data in detail.

In summary, interferometric calibration followed the recipes provided in the ALMA reduction scripts. Visual inspection of the different calibration steps (bandpass, phase, amplitude, and flux) showed that the scripts yielded a satisfactory calibration. For these data, calibration was performed within the Common Astronomy Software Application (CASA) versions 4.2.2 and 4.2.1 for the $12 \mathrm{~m}$ and $7 \mathrm{~m}$ array observations, respectively. We used the task statwt on calibrated science data to ensure that the weights were estimated in a consistent way for $7 \mathrm{~m}$ and $12 \mathrm{~m}$ data reduced with different versions of CASA ${ }^{2}$.

We imaged and post-processed the data using CASA version 5.4.0. The procedure is broadly implemented as follows. We spectrally regrid all data onto a common velocity frame with channel width $\sim 2.5 \mathrm{~km} \mathrm{~s}^{-1}$. Then we combine and jointly image all $12 \mathrm{~m}$ and $7 \mathrm{~m}$ data together. We deconvolve the data in two stages. First, we carry out a multi-scale clean using the CASA task tclean. We use a broad clean mask, and clean until the residual maps have a maximum signal-to-noise ratio of 4 . Then, we create a more restrictive clean mask and restart the cleaning using a single scale clean at the highest angular resolution in order to ensure that all point sources are completely deconvolved. We run this single scale clean until the flux in the model meets a convergence criterion related to the fractional change in the model flux during successive clean deconvolution cycles.

The total power data were reduced with CASA version 4.5.3 following a procedure described in Appendix A. Briefly, atmospheric and flux calibration, as well as the data gridding into a position-position-velocity cube, followed the recipes provided by ALMA. However, we did not use the baseline recipe delivered by ALMA. Instead, we fitted each individual spectrum before gridding with a polynomial of order 1 on the same baseline

\footnotetext{
2 See the CASA/ALMA documentation at https://casaguides nrao.edu/index.php/DataWeightsAndCombination
}

Table 1. Observational parameters of the ALMA observations.

\begin{tabular}{|c|c|c|c|c|c|}
\hline Line & $\begin{array}{c}v \\
{[\mathrm{GHz}]}\end{array}$ & $\begin{array}{c}\text { Noise } \\
{[\mathrm{K}]}\end{array}$ & $\begin{array}{c}\text { Restored } \\
\text { beam }\end{array}$ & $\begin{array}{c}\Delta v_{\text {res }} \\
{\left[\mathrm{km} \mathrm{s}^{-1}\right]}\end{array}$ & $\begin{array}{c}7 \mathrm{~m}+ \\
\mathrm{TP}\end{array}$ \\
\hline $\mathrm{CS}(5-4)$ & 244.94 & $0.12^{(a)}$ & $1^{\prime \prime} 0 \times 11^{\prime \prime} 0$ & 5.0 & No \\
\hline${ }^{12} \mathrm{CO}(2-1)$ & 230.54 & $0.17^{(a)}$ & $1 " 0 \times 1 " 0$ & 2.5 & Yes \\
\hline${ }^{12} \mathrm{CO}(1-0)$ & 115.27 & 0.12 & $1^{\prime \prime} .9 \times 3 " .1$ & 6.0 & Yes \\
\hline${ }^{13} \mathrm{CO}(1-0)$ & 110.20 & 0.03 & $2^{\prime \prime} 6 \times 3^{\prime \prime} .5$ & 6.0 & Yes \\
\hline $\mathrm{HNC}(1-0)$ & 90.66 & 1.2 & $2{ }^{\prime \prime} 0 \times 22^{\prime \prime} 1$ & 15.0 & No \\
\hline $\mathrm{HCO}^{+}(1-0)$ & 89.19 & 1.1 & $2^{\prime \prime} .0 \times 22^{\prime \prime} .2$ & 15.0 & No \\
\hline $\operatorname{HCN}(1-0)$ & 88.63 & 1.2 & $22^{\prime \prime} 1 \times 22^{\prime \prime} 2$ & 15.0 & No \\
\hline
\end{tabular}

Notes. ${ }^{(a)}$ Sensitivity measured in the region that was not fully covered by the interferometric data, which is where the headlight cloud is located. The sensitivity is 1.3 times better in the fully covered region.

window for all the spectra. This fixed baseline window covered the velocity ranges of $\left[0,590 \mathrm{~km} \mathrm{~s}^{-1}\right]$ and $\left[750,800 \mathrm{~km} \mathrm{~s}^{-1}\right]$, while the ALMA pipeline attempted to automatically adjust the velocity windows as a function of position. While this idea is appealing in principle, it is difficult to implement without a priori information on the source kinematics. In practice, it was biasing the baselining process by confusing low brightness signal with baseline noise. In our scheme we use our a priori information on the location of the galaxy signal in the velocity space.

After imaging, we converted the units of the cube to Kelvin, and primary beam corrected the data. The synthesized beam of the $12 \mathrm{~m}+7 \mathrm{~m}$ interferometric image is convolved with a Gaussian kernel to reach an angular full width at half maximum (FWHM) resolution of $1^{\prime \prime} 0$ (47 pc in linear scale at a distance of $9.6 \mathrm{Mpc}$ ). We then projected the single-dish data onto the astrometric grid of the interferometric image and used the CASA task feather to combine the two datasets.

We list the properties of the final cubes in Table 1. We note that the sensitivity of our final data cube is not homogeneous because a fraction of the $12 \mathrm{~m}$ and $7 \mathrm{~m}$ execution blocks did not cover the full field of view. A band of $\sim 40^{\prime \prime}$ width toward the northwestern edge thus has a noise 1.3 times higher than the remainder of the rectangular field of view. The headlight source lies within this band.

For CS(5-4), a simple single-resolution clean was applied to the $12 \mathrm{~m}$ data within the same mask as for the $\mathrm{CO}(2-1)$ emission. The $7 \mathrm{~m}$ and total power data were not added in this case because no emission was detected in these datasets. The deconvolved image was also convolved with a Gaussian to reach an angular resolution of 1 '. 0 .

\section{2. $3 \mathrm{~mm}$ lines}

\subsubsection{Observations}

Additional observations were acquired in Cycle 2 to image the emission from the $J=1-0$ transition of ${ }^{12} \mathrm{CO}$ (hereafter $\mathrm{CO}(1-0)),{ }^{13} \mathrm{CO}, \mathrm{C}^{18} \mathrm{O}, \mathrm{HCO}^{+}, \mathrm{HCN}$, and $\mathrm{HNC}$, as well as the $\mathrm{CS}(2-1)$ and $\mathrm{HNCO} 5(0,5)-4(0,4)$ lines. Three band-3 frequency setups at 90,110 , and $115 \mathrm{GHz}$ were required to cover all these emission lines. The first setup at $90 \mathrm{GHz}$ covers the 87.9-91.4 GHz (LSB) and 99.6-103.2 GHz (USB) frequency ranges. The second setup covers the $96-99.4 \mathrm{GHz}$ (LSB) and $108-111.7 \mathrm{GHz}$ (USB) ranges, and the third setup covers the $100.1-103.8 \mathrm{GHz}$ (LSB) and $112.3-115.5 \mathrm{GHz}$ (USB) ranges. The $110 \mathrm{GHz}$ and $115 \mathrm{GHz}$ setups target the $\mathrm{CO}$ lines that show both extended and compact emission. Thus, $7 \mathrm{~m}$ array and total 
power observations were performed in addition to the $12 \mathrm{~m}$ array observations in order to correctly recover the flux on all scales. Short-spacing observations for the $90 \mathrm{GHz}$ setup are available for NGC 628, but we chose not to incorporate them into the data cubes that we analyze here because the $90 \mathrm{GHz}$ setup targets lines mostly show compact emission structures.

The field of view covered by our observations at 90, 110, and $115 \mathrm{GHz}$ was $232^{\prime \prime} \times 170^{\prime \prime}, 240^{\prime \prime} \times 150^{\prime \prime}$, and $200^{\prime \prime} \times 150^{\prime \prime}$, respectively. Between 31 and $3812 \mathrm{~m}$ antennas and between 9 and $107 \mathrm{~m}$ antennas were used during the different observations. The total power observations used simultaneously either two or three antennas. The same OFF as for Cycle 1 was used during the on-the-fly observations.

\subsubsection{Reduction and imaging}

The data reduction recipes used for the $3 \mathrm{~mm}$ lines are much simpler than those used to reduce the $\operatorname{CO}(2-1)$. This is due to the fact that the $3 \mathrm{~mm}$ lines were reduced long before the recipes for the large program converged. In view of the satisfactory results obtained at relatively low signal-to-noise ratio, we did not try to refine them. This section describes these simple recipes.

Data calibration for the Cycle 2 band-3 data was performed in CASA, versions 4.2.1 and 4.3.1 for the interferometric data, and 4.4 and 4.5 for the total power data. Interferometric calibration followed the recipes provided in the ALMA reduction scripts because visual inspection showed that the scripts yielded a satisfactory calibration.

The $\mathrm{CO}$ and ${ }^{13} \mathrm{CO}(1-0)$ data were then spectrally smoothed to $6 \mathrm{~km} \mathrm{~s}^{-1}$. A signal mask was created from a $\mathrm{CO}(1-0)$ total power cube smoothed to an angular resolution of $60^{\prime \prime}$. The mask was created using the CPROPS signal detection algorithm: emission regions with a signal-to-noise ratio higher than 3 were identified and then extended to include contiguous pixels with a signal-to-noise ratio higher than 2 . This mask was used to guide the deconvolution of the $\mathrm{CO}$ and ${ }^{13} \mathrm{CO}(1-0)$ data.

A single-scale clean algorithm, as coded in the CASA task tclean, was applied to the $12 \mathrm{~m}+7 \mathrm{~m}$ data up to the point where the residual maximum fell below $1 \mathrm{mJy}$. The total power cube was used as an initial model. The resulting cleaned cube was then feathered with the total power cube.

The fainter lines were imaged at coarser spatial and spectral resolution (see Table 1 for details). Total power cubes were not used during the deconvolution in these cases (neither as models, nor for short-spacing correction) as the signal was barely or not at all detected in the single-dish observations.

\subsection{Complementary MUSE data}

We use MUSE observations to characterize the ionized gas and stellar content associated with the headlight cloud. MUSE is an optical IFU on the VLT in Paranal, Chile. Kreckel et al. (2016, 2018) present a detailed explanation of the strategy and data reduction of the MUSE observations of NGC 628. We summarize a few key aspects here.

The observations cover a wavelength range from 4800 to $9300 \AA$, which includes the $\mathrm{H} \alpha$ and $\mathrm{H} \beta$ emission lines and a large region of the optical stellar continuum. The velocity resolution is about $150 \mathrm{~km} \mathrm{~s}^{-1}$. In the mode that we used, the field of view of the IFU is $1^{\prime} \times 1^{\prime}$ with $0^{\prime \prime} .2$ pixels. We paneled multiple fields of view to cover the whole inner part of the galaxy (see Kreckel et al. 2018). The seeing of the observations was $\sim 1^{\prime \prime}\left(\sim 47 \mathrm{pc}\right.$ in linear scale) with an astrometric accuracy of $00^{\prime \prime} 2$.
The $\mathrm{H} \alpha$ emission line was obtained using the LZIFU pipeline (Ho et al. 2016), which simultaneously fits and subtracts the stellar continuum. All line fluxes quoted in this paper were corrected for extinction. The emission line reddening was inferred by comparing the observed $\mathrm{H} \alpha$ to $\mathrm{H} \beta$ line ratio to an intrinsic value of 2.86 (for case $\mathrm{B}$ recombination and an electron temperature of $10000 \mathrm{~K}$ ) under the assumption of a Fitzpatrick (1999) extinction curve with $R_{V}=3.1$. Typical values for $E(B-V)$ are $\sim 0.5$, including for the headlight cloud.

\section{Molecular gas in the headlight cloud}

\section{1. $\mathrm{CO}(2-1)$ emission}

The top row of Fig. 1 presents ALMA maps of the $\mathrm{CO}(2-1)$ peak brightness and integrated intensity across NGC 628, saturated to high intensity of $3.6 \mathrm{~K}$ and $45 \mathrm{~K} \mathrm{~km} \mathrm{~s}^{-1}$, respectively. This is a compromise to show the spatial distribution of the $\sim 99.99 \%$ of the pixels even though this may give the incorrect impression that other clouds in NGC 628 are as bright as the headlight cloud. The headlight cloud is located within an outer spiral arm, at an offset of [47", 51"] and a radial distance of $3.2 \mathrm{kpc}$ from the galaxy center. The headlight cloud is visible as the brightest peak in both panels of Fig. 1.

In the bottom row of Fig. 1, we plot pixel-wise cumulative distribution functions (CDFs) of the $\mathrm{CO}(2-1)$ peak brightness $T_{\mathrm{CO}}$ and the integrated intensity $W_{\mathrm{CO}}$ across the ALMA field of view. The CDF is a one-point statistic that quantifies how the emission in each map is distributed between low- and highbrightness regions (for a detailed discussion of $\mathrm{CO}$ distribution functions see Hughes et al. 2013).

The headlight cloud stands out in both CDFs. For both $T_{\mathrm{CO}}$ and $W_{\mathrm{CO}}$, the distributions clearly show a change in slope for the 100 brightest pixels on the map, which we indicate with a red line. The sense is that above a high threshold, there is more bright $\mathrm{CO}$ emission than would be predicted based on the rest of the galaxy; that is, the slope of the distribution function becomes flatter. All of these unusually bright pixels above either threshold belong to a region surrounding the headlight cloud.

This bright emission is notable for both its shape and its location. We constructed equivalent CDFs for the peak brightness and integrated intensity maps of the $\mathrm{CO}(1-0)$ emission measured by the PAWS survey of M 51 (Schinnerer et al. 2013; Pety et al. 2013) and the MAGMA survey of the Large Magellanic Cloud (LMC; Wong et al. 2011), and of CO(2-1) emission measured by the IRAM $30 \mathrm{~m}$ survey of M 33 (Druard et al. 2014), finding no similar features (see also Hughes et al. 2013, but we note that this paper uses the probability distribution function rather than CDF formalism). In the mass distribution functions plotted by Sun et al. (2018, including NGC 628), bumps and features at high brightness are almost always associated with galaxy centers or dynamical environments like stellar bars. The headlight cloud lies in the outer part of a spiral arm, not associated with either the galaxy center or a stellar bar.

In Fig. 2 we zoom in on the headlight cloud. These maps show the $\mathrm{CO}(2-1)$ peak brightness, integrated intensity, centroid velocity, and FWHM in a large region around the cloud (left) and in the immediate vicinity of the cloud (right). They show highly concentrated $\mathrm{CO}$ emission, with a compact bright peak $\left(T_{\text {peak }}>6 \mathrm{~K}\right.$ over 1 square arcsecond $\left.\sim 2000 \mathrm{pc}^{2}\right)$. This bright peak is surrounded by a more extended component with $T_{\text {peak }}>$ $1 \mathrm{~K}$ over $\sim 50$ square arcseconds or $\sim 100000 \mathrm{pc}^{2}$.

Morphologically, the cloud appears to be linked to other material in the spiral arm by four filaments. A fifth filament 

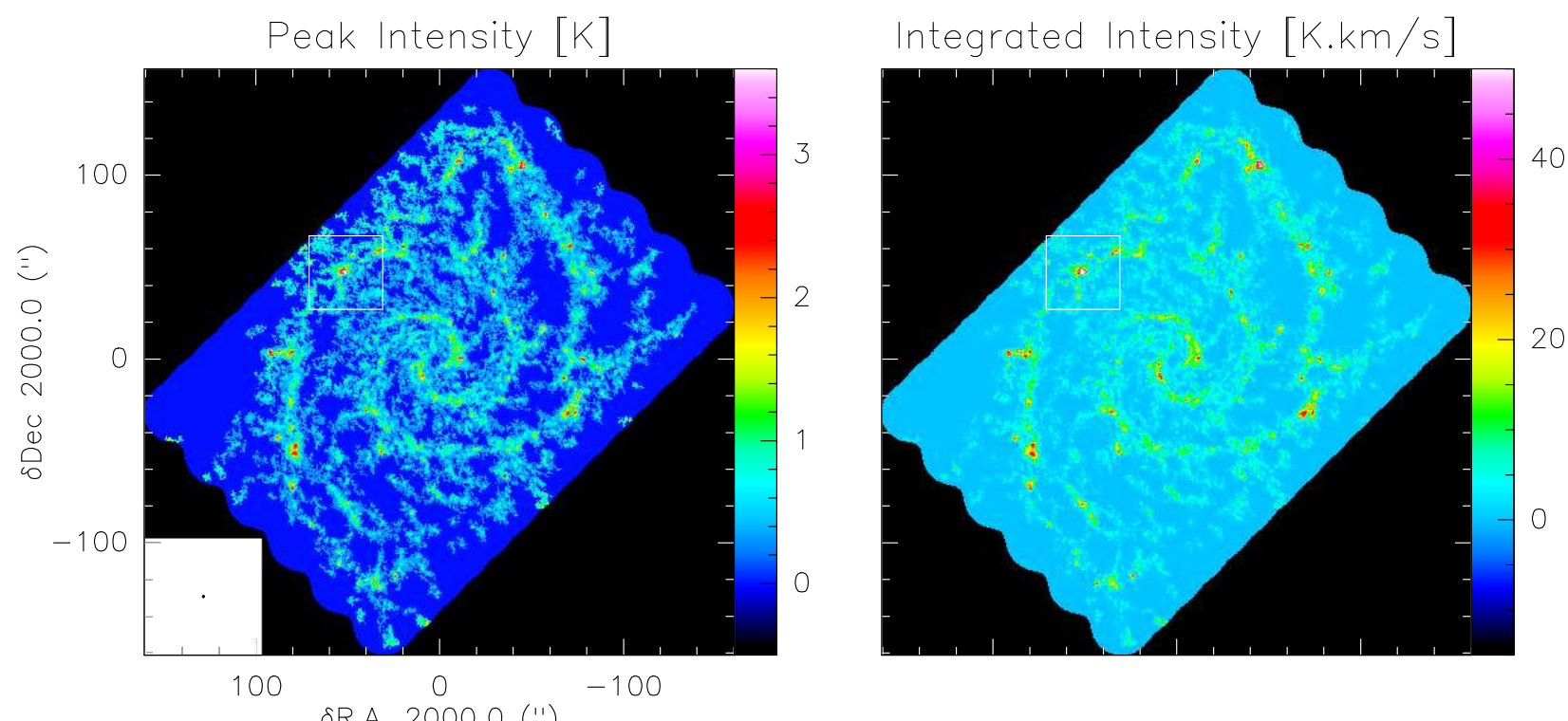

SR.A. 2000.0 (')

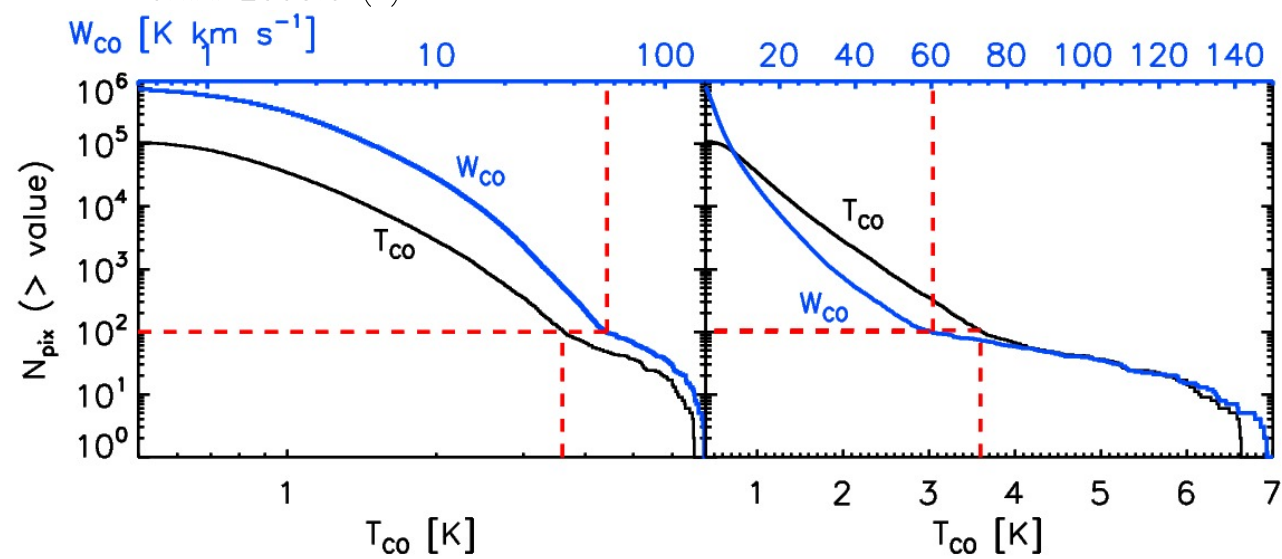

Fig. 1. Top: spatial distribution of the peak temperature (left) and integrated intensity (right) of the $\mathrm{CO}(2-1)$ line over the full observed field of view of NGC 628. The color scales a dynamic range of the peak temperature and integrated intensity, saturated to high intensity of $3.6 \mathrm{~K}$ and $45 \mathrm{~K} \mathrm{~km} \mathrm{~s}^{-1}$, respectively. The white square shows the position of the headlight cloud in NGC 628 (see the left part of Fig. 2 for a magnification). Offset positions are from the galactic center, $\alpha: 01^{\mathrm{h}} 36^{\mathrm{m}} 41 \mathrm{~s} 72, \delta:+15^{\circ} 46^{\prime} 59^{\prime \prime} 3 \mathrm{~J} 2000$. The beamsize is shown in the bottom left corner of the left panel. Bottom: number of pixels whose $\mathrm{CO}(2-1)$ peak temperature $T_{\mathrm{CO}}$ (black curve) and integrated $\mathrm{CO}(2-1)$ intensity $W_{\mathrm{CO}}$ (blue curve) are above the value given in the bottom abscissa for $T_{\mathrm{CO}}$ and in the top abscissa for $W_{\mathrm{CO}}$, for the entire galaxy. Shown is the full range of values in logarithmic scale (left) and the zoomed-in linear scale $\left(\right.$ right). The red dashed lines show the values corresponding to 100 pixels (or a surface of about $\left.2^{\prime \prime} \times 2^{\prime \prime}\right)$ in the CO map.

extends southward from the cloud into the interarm region. The velocity field within the cloud, as traced by the centroid velocity, is relatively smooth, while the gas in the southern filament appears shifted to higher velocities compared to the cloud material.

The gas velocity dispersion in the cloud is high, and increases toward the cloud center: within $\sim 2$ arcsec of the intensity peak, the FWHM linewidths are $>15 \mathrm{~km} \mathrm{~s}^{-1}$ with a maximum value of $24 \mathrm{~km} \mathrm{~s}^{-1}$. Typical $\mathrm{CO}(2-1)$ FWHM linewidths in the surrounding spiral arm are $\leq 10 \mathrm{~km} \mathrm{~s}^{-1}$ (see Fig. 2 top right panels).

\subsection{GMC properties in NGC 628}

Catalogs of giant molecular clouds (GMCs) for all the PHANGS-ALMA galaxies, including NGC 628, have been constructed and will be presented in a forthcoming paper (Rosolowsky et al., in prep.). These catalogs are generated using the CPROPS algorithm (Rosolowsky \& Leroy 2006) to segment the $\mathrm{CO}(2-1)$ data cubes into individual molecular clouds and then measure the radius, linewidth, luminosity, and other structural properties of each cloud.

\subsubsection{The headlight cloud}

The cataloged radius and (FWHM) linewidth of the headlight cloud are $184 \mathrm{pc}$ and $16.3 \mathrm{~km} \mathrm{~s}^{-1}$, respectively. The measured radius of $184 \mathrm{pc}$ has been deconvolved by the beam size, extrapolated to correct for sensitivity and blending effects, and then converted to the radius convention defined by Solomon et al. (1987), which implies multiplying the rms size by 1.91 (for details see Rosolowsky \& Leroy 2006). For a Gaussian shape, this implies a cloud FWHM size of $\sim 220$ pc.

The headlight cloud is the most luminous of the 850 clouds in the catalog, with a $\mathrm{CO}$ luminosity mass of $M_{\mathrm{lum}}=\alpha_{\mathrm{CO}} L_{\mathrm{CO}}=$ $2.0 \times 10^{7} M_{\odot}$. This mass assumes a $\mathrm{CO}(2-1)$-to- $\mathrm{H}_{2}$ conversion factor $\alpha_{\mathrm{CO}}=6.2 M_{\odot}\left(\mathrm{K} \mathrm{km} \mathrm{s}^{-1} \mathrm{pc}^{2}\right)^{-1}$, which is the Galactic $\mathrm{CO}(1-0)-$ to- $\mathrm{H}_{2}$ conversion factor, $4.3 M_{\odot}\left(\mathrm{K} \mathrm{km} \mathrm{s}^{-1} \mathrm{pc}^{2}\right)^{-1}$ 


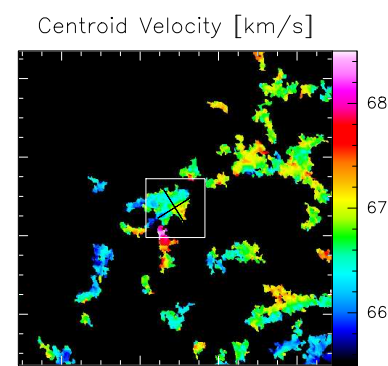

Peak Intensity $[K]$

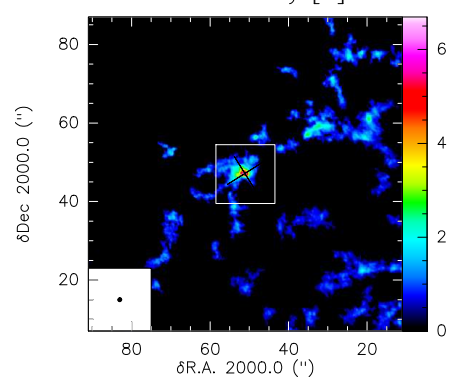

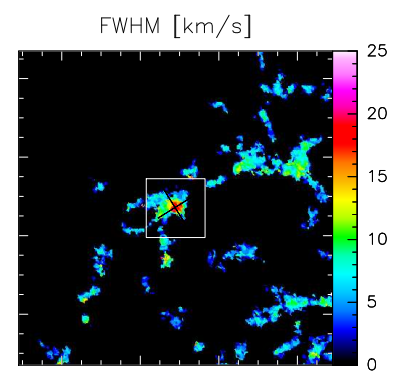

Integrated Intensity $[\mathrm{K} . \mathrm{km} / \mathrm{s}]$

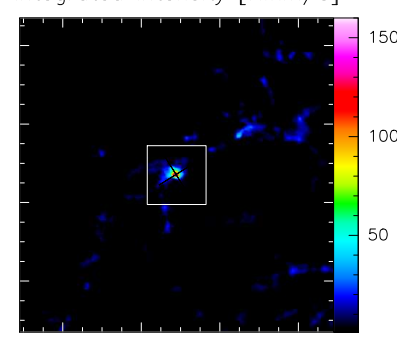

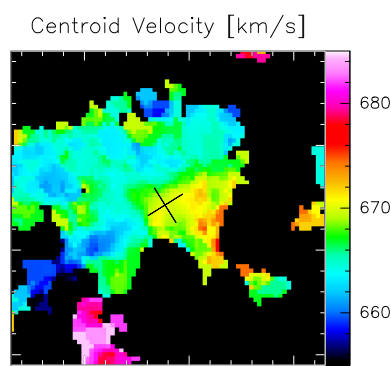

Peak Intensity $[K]$

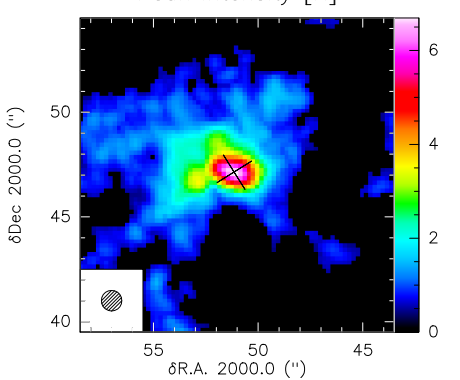

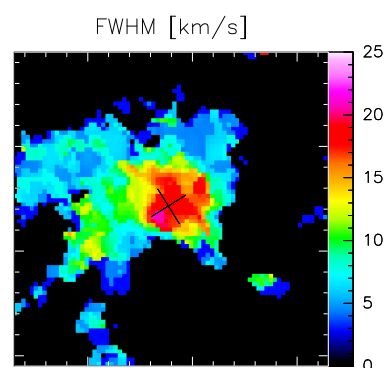

Integrated Intensity $[\mathrm{K} . \mathrm{km} / \mathrm{s}]$

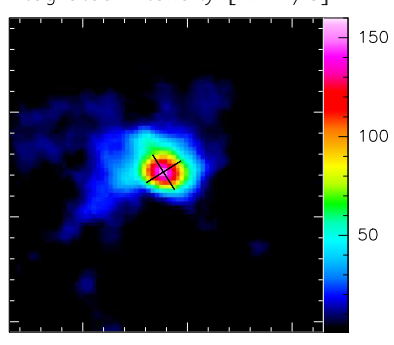

Fig. 2. Spatial distributions of the moments of the $\mathrm{CO}(2-1)$ line centered over the headlight cloud. The four left panels show how this molecular cloud relates to the spiral arm, covering a field of view of $80^{\prime \prime} \times 80^{\prime \prime}$, while the four right panels zoom in on the molecular cloud itself. The beamsize is shown in the bottom left corner of the bottom left panels.

Table 2. Physical properties of the ten most CO luminous clouds measured by CPROPS, sorted by decreasing luminous mass.

\begin{tabular}{|c|c|c|c|c|c|c|c|c|c|c|c|}
\hline \multirow[b]{2}{*}{$\begin{array}{l}\text { Cloud } \\
\text { ID }\end{array}$} & \multicolumn{10}{|c|}{ Molecular gas } & \multirow{2}{*}{$\begin{array}{c}\text { H II region }^{(\dagger)} \\
L_{\mathrm{H} \alpha} \\
\mathrm{erg} \mathrm{s}^{-1}\end{array}$} \\
\hline & $\begin{array}{c}\text { RA } \\
\text { h:m:s }\end{array}$ & $\begin{array}{c}\text { Dec } \\
\circ:{ }^{\prime}: " .\end{array}$ & $\begin{array}{c}M_{\mathrm{lum}} \\
10^{5} M_{\odot} \\
\end{array}$ & $\begin{array}{c}M_{\mathrm{vir}} \\
10^{5} M_{\odot} \\
\end{array}$ & $\begin{array}{c}\text { Rad. } \\
\text { pc }\end{array}$ & $\begin{array}{c}\Delta v \\
\mathrm{~km} \mathrm{~s}^{-1}\end{array}$ & $\begin{array}{c}\alpha_{\text {vir }} \\
- \\
\end{array}$ & $\begin{array}{c}\Sigma_{\mathrm{mol}} \\
M_{\odot} \mathrm{pc}^{-2}\end{array}$ & $\begin{array}{c}R_{21} \\
- \\
\end{array}$ & $\begin{array}{c}D_{\text {corot }} \\
\mathrm{kpc}\end{array}$ & \\
\hline $\mathrm{L} 1^{(\star)}$ & $01: 36: 45.328$ & $15: 47: 48.48$ & 204 & 91 & 184 & 16.3 & 0.5 & 192 & 0.70 & 0.00 & $6.3 \times 10^{39}$ \\
\hline $\mathrm{L} 2$ & $01: 36: 41.757$ & $15: 47: 22.17$ & 149 & 92 & 280 & 13.2 & 0.7 & 61 & 0.61 & 1.71 & $5.5 \times 10^{36}$ \\
\hline L3 & $01: 36: 47.144$ & $15: 46: 08.77$ & 142 & 73 & 193 & 14.2 & 0.6 & 121 & 0.71 & 0.37 & $7.1 \times 10^{38}$ \\
\hline L4 & $01: 36: 38.574$ & $15: 48: 46.11$ & 139 & 68 & 141 & 16.0 & 0.5 & 223 & $-(\dagger \dagger)$ & 0.00 & $8.2 \times 10^{38}$ \\
\hline L5 & $01: 36: 42.337$ & $15: 46: 50.22$ & 127 & 131 & 246 & 16.9 & 1.2 & 66 & 0.60 & 2.18 & $2.6 \times 10^{37}$ \\
\hline L6 & $01: 36: 42.974$ & $15: 47: 23.85$ & 118 & 83 & 302 & 12.1 & 0.8 & 41 & 0.60 & 1.35 & $8.6 \times 10^{36}$ \\
\hline L7 & $01: 36: 44.038$ & $15: 46: 35.88$ & 118 & 172 & 355 & 16.1 & 1.6 & 30 & 0.56 & 0.87 & $4.2 \times 10^{36}$ \\
\hline L8 & $01: 36: 36.942$ & $15: 46: 31.21$ & 105 & 63 & 145 & 15.2 & 0.7 & 159 & 0.61 & 0.00 & $6.6 \times 10^{37}$ \\
\hline L9 & 01:36:43.859 & $15: 48: 01.02$ & 88 & 52 & 204 & 11.6 & 0.7 & 67 & 0.57 & 0.00 & $1.3 \times 10^{37}$ \\
\hline L10 & 01:36:41.309 & $15: 46: 59.40$ & 86 & 89 & 212 & 15.0 & 1.2 & 61 & 0.63 & 2.50 & $7.7 \times 10^{36}$ \\
\hline NGC 628 Mean & - & - & 14 & 22 & 91 & 10.3 & 2.1 & 56 & 0.54 & - & - \\
\hline
\end{tabular}

Notes. The last line lists the parameters averaged in all molecular clouds in NGC 628. From Cols. 1 to 11: cloud identification, cloud coordinates in RA and Dec, luminous CO mass, virial mass, radius, velocity linewidth, virial parameter, molecular gas mass surface density, $\mathrm{CO}(2-1) / \mathrm{CO}(1-0)$, and measured distance to the closest corotation region. Column 12 lists the $\mathrm{H} \alpha$ luminosity of the associated $\mathrm{H}$ II region estimated from MUSE observations from Kreckel et al. (2017). ${ }^{(\star)}$ Cloud 1 corresponds to the NGC 628 headlight. ${ }^{(\dagger)}$ Often there are multiple objects within a 150 pc region. We list the closest $\mathrm{H}$ II region. ${ }^{(\dagger)}$ Cloud L4 falls just outside the coverage of the $\mathrm{CO}(1-0)$ observations.

(Bolatto et al. 2013), divided by 0.69 (i.e., the $\mathrm{CO}(2-1) / \mathrm{CO}(1-0)$ ratio measured in the headlight cloud; see Sect. 6.1). This $\mathrm{CO}(2-1) / \mathrm{CO}(1-0)$ value is close to the nearby galaxy canonical value of 0.7 (e.g., Leroy et al. 2009, 2013; Saintonge et al. 2017) and to the mean ratio measured on kiloparsec scales in NGC 628 by the recent EMPIRE survey (Jiménez-Donaire et al. 2019, see also Table 5).

Treating the geometry as a three-dimensional Gaussian, the measured mass and radius of the headlight cloud imply an $\mathrm{H}_{2}$ density of $n_{\mathrm{H}_{2}} \sim 20 \mathrm{~cm}^{-3}$. This is considerably lower than the critical density of $\mathrm{CO}(2-1)$ emission, even allowing for line trapping, and implies significant clumping or substructure within the cloud. The bulk physical parameters of the headlight cloud correspond to free-fall time and Mach number typical of massive giant molecular clouds. Using a mean density $n_{\mathrm{H}_{2}} \sim 20 \mathrm{~cm}^{-3}$, the free-fall time $t_{\mathrm{ff}}=\sqrt{3 \pi /(32 G \rho)}$ and crossing time $t_{\text {cross }}=R / \Delta v$ are 8 and $11 \mathrm{Myr}$ respectively. Assuming a gas temperature of $\sim 20 \mathrm{~K}$ (see Sect. 3.3.3), the measured linewidth corresponds to a Mach number of $\sim 42$.

\subsubsection{Comparison with other luminous clouds in NGC 628}

To place these values in context, Table 2 reports the physical properties (mass, radius, virial parameter) of the ten most luminous GMCs in NGC 628, as well as the mean value for the entire cataloged GMC population of NGC 628. We compare the headlight cloud to the full GMC population and highlight these massive clouds in Fig. 3.

The $184 \mathrm{pc}$ radius of the headlight cloud is large, but does not clearly distinguish the cloud from other massive clouds 

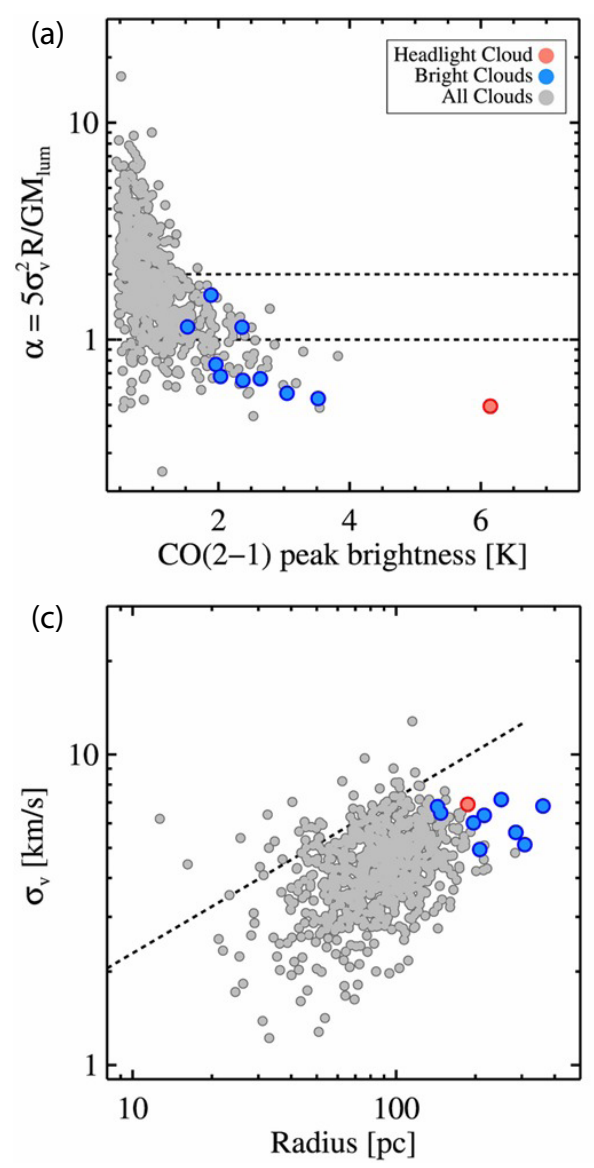
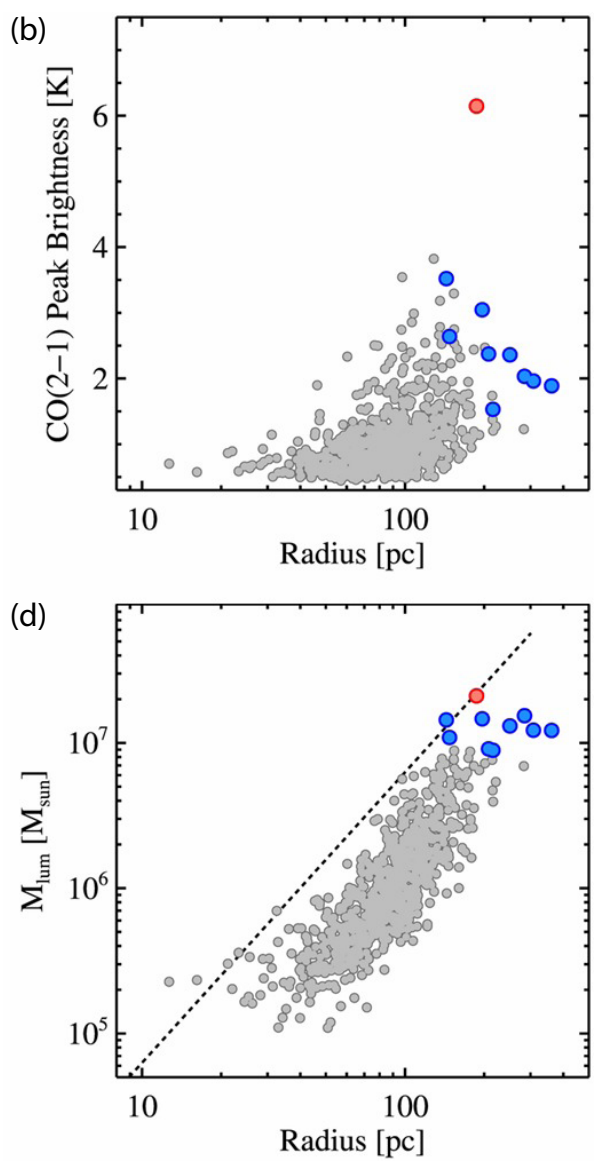

Fig. 3. Scaling relations between physical properties of the molecular clouds in NGC 628: (a) virial parameter as a function of $\mathrm{CO}$ peak brightness, (b) $\mathrm{CO}$ peak brightness as a function of cloud size, $(c)$ linewidth as a function of size, and $(d)$ luminous mass as a function of size. In all panels the headlight cloud is the red point, and the nine next most luminous $\left(M_{\text {lum }}>8.6 \times\right.$ $10^{6} M_{\odot}$ ) molecular clouds are blue. The horizontal dashed lines in the top left panel represent virial parameters of $\alpha=1$ and $\alpha=2$, values that are indicative of virialized and self-gravitating clouds, respectively. The dashed line in the bottom left panel represents the size-linewidth relation reported by Solomon et al. (1987) for inner Milky Way clouds. The dashed line in the bottom right panel represents a mass surface density of $\Sigma_{\text {cld }}=200 M_{\odot} \mathrm{pc}^{-2}$. in NGC 628. The global linewidth of the headlight cloud $\left(16.3 \mathrm{~km} \mathrm{~s}^{-1}\right)$ is also large but similar to that of the other high-mass GMCs in NGC 628. As a result, in the bottom left panel of Fig. 3, the headlight cloud clusters with the other massive clouds at the high end of the linewidth-size relation.

In contrast, the headlight cloud stands out in surface density. The bottom right panel of Fig. 3 shows the luminous mass as a function of size. The dashed line represents a mass surface density of $\Sigma_{\text {cld }} \sim 200 M_{\odot} \mathrm{pc}^{-2}$. The mass surface density of the headlight cloud, estimated from the luminous mass, is higher than any other massive cloud but one and is among the highest in the galaxy. This value, $\Sigma_{\mathrm{CO}} \sim 190 M_{\odot} \mathrm{pc}^{-2}$, is roughly three times greater than the average value for all GMCs in NGC 628.

The most exceptional property of the $\mathrm{CO}(2-1)$ emission in the headlight cloud is its peak brightness (i.e., the intensity of the brightest pixel in the cloud at the $\sim 1^{\prime \prime}$ resolution of our PHANGS-ALMA $\mathrm{CO}(2-1)$ data). In the top row of Fig. 3 the headlight cloud (in red) clearly separates from the other massive clouds (in blue), with a peak brightness of almost $7 \mathrm{~K}$. This is roughly twice the peak brightness of the other massive clouds and the highest value found for any cloud in the galaxy.

A consequence of the headlight cloud's high surface density and high brightness is that the cloud appears tightly bound. The virial parameter of the headlight cloud, estimated from $\alpha_{\text {vir }}=5 \sigma_{\mathrm{v}}^{2} R / G M_{\text {lum }}$, is low, 0.5. This is low compared to both most other clouds in the galaxy and the value expected for virialized $\left(\alpha_{\mathrm{vir}}=1\right)$ or marginally bound $\left(\alpha_{\mathrm{vir}}=2\right)$ GMCs. We note that the low virial parameter of the headlight cloud (and many of the other massive clouds) appears to be part of a systematic trend in NGC 628 for the GMC virial parameters to decrease with increasing peak brightness (top left panel of Fig. 3) and $M_{\text {lum }}$ (not shown). This trend needs to be interpreted with care: the scatter at low brightness is partly due to the impact of marginal resolution on these measurements, while $\mathrm{CO}$ brightness also enters in the denominator of the virial parameter via the definition of $M_{\text {lum }}$. Nevertheless, simulations do predict mass- and environment-dependent variations in cloud virialization (e.g., Federrath \& Klessen 2012), and we plan to investigate these trends in more detail using the full PHANGS-ALMA sample in a future paper.

Figure 3 shows that the headlight cloud has a large size and velocity dispersion like the other massive clouds in NGC 628, but an exceptionally bright core. This implies a unique profile for the cloud within the NGC 628 GMC population. Figure 4 examines this profile in more detail. The top left panel plots the $\mathrm{CO}(2-1)$ integrated intensity as a function of distance away from the brightest pixel in the cloud for each of the ten most massive GMCs in NGC 628. The top right panel shows the same, but normalizing all clouds by the integrated intensity at the cloud center. The bottom row of Fig. 4 is similar, but here we plot radial profiles of the $\mathrm{CO}(2-1)$ linewidth instead of integrated intensity. We use the second moment to parameterize the cloud linewidth in Fig. 4, but a similar result is obtained using other linewidth diagnostics.

Figure 4 shows that the headlight cloud has the brightest core among all the massive clouds, being more than twice as bright as the next brightest massive cloud. The core is surrounded by an extended envelope of lower intensity emission, which leads to the large size estimated by CPROPS. The top right panel shows that in relative terms the headlight cloud has the most compact 

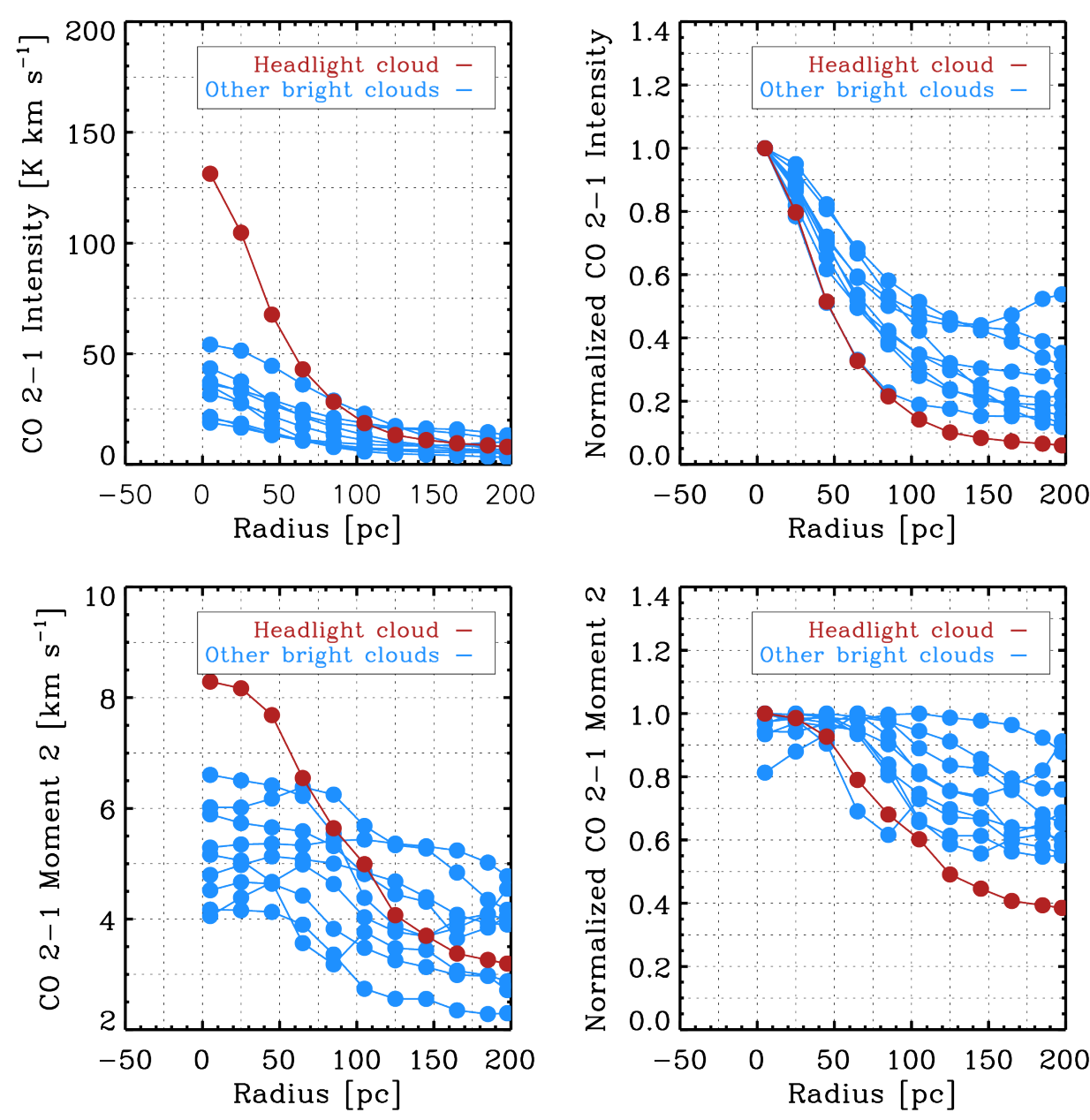

Fig. 4. Profiles of mean integrated $\mathrm{CO}(2-1)$ intensity (top row) and mean $\mathrm{CO}(2-1)$ linewidth (bottom row) around the ten most luminous clouds in NGC 628, with the headlight cloud highlighted in red. The second moment is used to characterize the linewidth. For each cloud the azimuthally averaged quantity in radial bins centered on the peak of the cloud is measured. Right panels: radial profiles normalized by the value at the central position of each cloud.

profile of all massive clouds in NGC 628, though still moderately extended compared to the beam. The velocity profile of the headlight cloud is also distinctive, with an enhanced central linewidth and a steeper decrease in the line width between the center and edge of the cloud.

Figures 3 and 4 thus tend to reinforce the visual impression from Fig. 1 that the $\mathrm{CO}$ emission associated with the headlight cloud has exceptional properties. After integrating over the extent of the CPROPS-identified cloud, the headlight cloud resembles other massive clouds in NGC 628 in terms of size, line width, and mass, yet looking more closely it stands out due to its compact profile and enhanced central linewidth. Below, we show that the headlight cloud's compact CO-bright core is coincident with a bright $\mathrm{H}$ II region. This, combined with its apparently strong self-gravity, indicates a massive star-forming region caught in the early stages of forming stars, and perhaps a potential birth site of massive clusters.

\subsection{Other molecular emission lines}

In addition to $\mathrm{CO}(2-1)$, we observed NGC 628 in $\mathrm{CO}(1-0)$, ${ }^{13} \mathrm{CO}(1-0), \mathrm{CS}(5-4), \mathrm{HCO}^{+}(1-0), \mathrm{HCN}(1-0)$, and $\mathrm{HNC}(1-0)$. The ratios among these lines allow us to further constrain the physical conditions (e.g., density, temperature) within the headlight cloud.

\subsubsection{Observed line ratios in the headlight cloud}

The headlight cloud is detected in all molecular tracers except $\mathrm{CS}(5-4)$. Moreover, it is the only source detected in these trac- ers in the $12 \mathrm{~m}$ ALMA observations. The locations of the emission peaks for the different molecular lines match to within $<1^{\prime \prime}$ for the $\mathrm{CO}$ lines and to within 1'!8 for the high-density tracers. These higher density tracers also have low signal-to-noise ratios (see Fig. B.1). As a result, it remains unclear whether the offsets are significant. In the remainder of this article, we consider that the peaks for all lines are spatially coincident within our current measurement accuracy. As a consequence, the left panel in Fig. 5 shows the line profiles extracted at the position of the peak emission of each molecular line. To make a fair comparison, we first smooth all images to match the resolution of the coarsest resolution dataset (i.e., ${ }^{13} \mathrm{CO}(1-0)$ ) before extracting the line profile at the emission peak (see Fig. B.2).

We fit a single Gaussian profile to each line in Fig. 5 and report the results in Table 3 . We do not include the values for HNC $(1-0)$ since no reliable fit could be obtained. The centroid velocities and linewidths of all tracers are in good agreement, although HCN appears somewhat broader than the other lines. We have verified that this is not due to the hyper-fine structure of the $\mathrm{HCN}(1-0)$ line, but it may be an artifact of the limited velocity resolution and sensitivity of the HCN data. Table 4 lists the peak brightness and integrated intensity line ratios for different combinations of the emission lines. We use these ratios in Sect. 3.3.3 to derive an estimate of the density and temperature of the molecular gas in the headlight cloud.

\subsubsection{Comparison to literature line ratios}

Here we compare the line ratios that we measure for the headlight cloud to a compilation of line ratios in other Galactic 

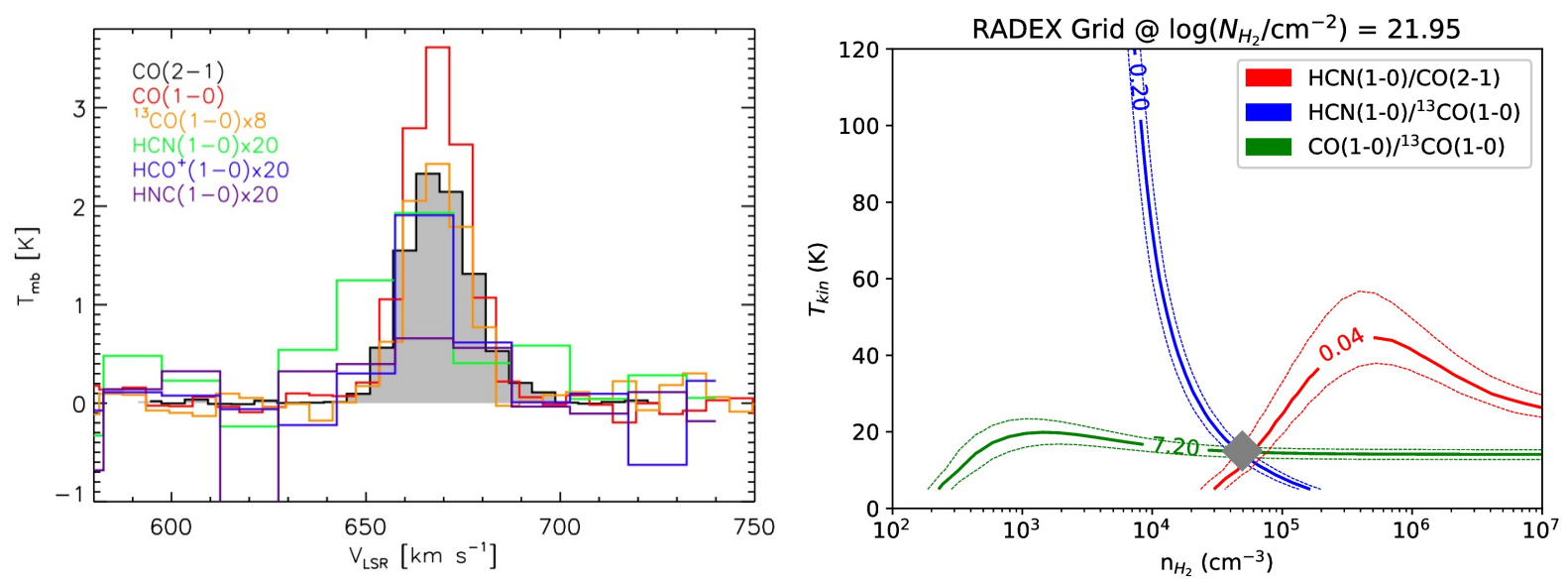

Fig. 5. Left: line profiles of all measured molecular gas tracers extracted at the position of the peak emission of each line, after convolving all data to the angular resolution of ${ }^{13} \mathrm{CO}(1-0)$. The $\mathrm{CO}(2-1)$ spectrum appears as the gray filled region. Other lines are scaled by the factors indicated in the legend to put them on the same plot. Right: RADEX output showing fits to the observed ratios among these lines. Blue, red and green lines correspond to the observed $\mathrm{HCN} /{ }^{13} \mathrm{CO}, \mathrm{HCN}(1-0) / \mathrm{CO}(2-1)$, and ${ }^{12} \mathrm{CO} /{ }^{13} \mathrm{CO}$ values. The gray diamond indicates the position on the RADEX $\left[n_{\mathrm{H}_{2}}, T_{\text {kin }}\right]$ grid where the $\chi^{2}$ is minimal (i.e., the best-fit values). The dashed lines show the $95 \%$ confidence level of the $\chi^{2}$ fit, which is smaller than the size of the diamond.

Table 3. Best-fit parameters of the molecular line profiles.

\begin{tabular}{lccc}
\hline \hline & \multicolumn{3}{c}{ At peak, convolved to ${ }^{13} \mathrm{CO}$ beam } \\
\hline Line & $\begin{array}{c}T_{\text {Peak }} \\
{[\mathrm{K}]}\end{array}$ & $\begin{array}{c}V_{\mathrm{LSR}} \\
{\left[\mathrm{km} \mathrm{s}^{-1}\right]}\end{array}$ & $\begin{array}{c}\Delta v \\
{\left[\mathrm{~km} \mathrm{~s}^{-1}\right]}\end{array}$ \\
\hline $\mathrm{CO}(2-1)$ & $2.52 \pm 0.02$ & $668.3 \pm 0.1$ & $19.5 \pm 0.2$ \\
$\mathrm{CO}(1-0)$ & $3.64 \pm 0.11$ & $668.3 \pm 0.3$ & $18.1 \pm 0.6$ \\
${ }^{13} \mathrm{CO}(1-0)$ & $0.51 \pm 0.02$ & $668.2 \pm 0.4$ & $17.7 \pm 0.9$ \\
$\mathrm{HCO}^{+}(1-0)$ & $0.10 \pm 0.01$ & $666.8 \pm 1.8$ & $20.4 \pm 3.0$ \\
$\mathrm{HCN}(1-0)$ & $0.09 \pm 0.02$ & $660.4 \pm 2.7$ & $32.2 \pm 6.5$ \\
\hline
\end{tabular}

Notes. The line profiles are extracted at the position of the peak of the headlight cloud after convolution to a common angular resolution. The quoted uncertainties in the parameters correspond to the errors in the Gaussian fits, and the FWHM is reported as $\Delta v$. At $9.6 \mathrm{Mpc}$, the $3^{\prime \prime} .5 \times$ 2 ".6 Gaussian beam of the matched resolution input data corresponds to a physical area of $\sim 22300 \mathrm{pc}^{2}$.

and extragalactic sources. We compared to nine nearby spiral galaxies that are part of the EMPIRE survey (Bigiel et al. 2016) using the values determined by Cormier et al. (2018) and Jiménez-Donaire et al. (2019). We also compared to the line ratios measured in Orion B in the Milky Way (Sakamoto et al. 1994; Pety et al. 2017) and 30 Doradus in the LMC (Johansson et al. 1998; Pineda et al. 2012; Anderson et al.2014). Table 5 lists these ratios, the associated spatial scales, and the references for the measurements.

Figure 6 shows the $\mathrm{HCN} / \mathrm{CO}(1-0)$ value in green, $\mathrm{HCO}^{+} /$ $\mathrm{HCN}$ in red, ${ }^{13} \mathrm{CO} /{ }^{12} \mathrm{CO}$ in yellow, and $\mathrm{CO}(2-1) / \mathrm{CO}(1-0)$ in blue. In each case, we plot the line ratio in the comparison source normalized to the value estimated for the headlight cloud, so that it indicates a line ratio identical to the headlight cloud. From the top to bottom panel, we plot the data for entire galaxies, and for disk and center regions separately. We do not show data for the inter-arm or diffuse extended regions. This is why the galactic average values can sometimes be lower than both the disk and center values. The line ratios used in this plot and quoted in Table 5 were derived using different methods and on datasets with a range of resolutions and sensitivities, and the values should therefore be interpreted with some caution. As described in Sect. 3.3, the values for the headlight cloud were
Table 4. Line ratios at the peak of the headlight cloud.

\begin{tabular}{ccc}
\hline \hline Ratio & Peak & Integrated \\
\hline$\frac{\mathrm{CO}(2-1)}{\mathrm{CO}(1-0)}$ & 0.70 & 0.75 \\
$\frac{{ }^{12} \mathrm{CO}(1-0)}{13 \mathrm{CO}(1-0)}$ & 7.2 & 7.3 \\
$\frac{\mathrm{HCO}^{+}(1-0)}{\mathrm{HCN}^{1}(1-0)}$ & 1.1 & 0.7 \\
$\frac{\mathrm{HCO}^{+}(1-0)}{12} \mathrm{CO}(1-0)$ & 0.028 & 0.031 \\
$\frac{\mathrm{HCN}(1-0)}{12 \mathrm{CO}(1-0)}$ & 0.025 & 0.043 \\
$\frac{\mathrm{HCN}(1-0)}{{ }^{13} \mathrm{CO}(1-0)}$ & 0.2 & 0.3 \\
\hline
\end{tabular}

Notes. The line ratios are calculated using (i) the peak brightness and (ii) the integrated intensity of each emission line, as determined by a Gaussian fit to the line profile (see Table 3). The line profiles are extracted at the position of the peak of the headlight cloud after convolution to a common angular resolution.

determined using the peak brightness of a Gaussian fit to line profiles extracted at a spatial scale of $\sim 140 \mathrm{pc}$, whereas the values for the EMPIRE galaxies are ratios calculated using the integrated intensity of an average line profile from within a kiloparsec scale aperture. A comparison between ratios measured at similar scales will only be possible when more high angular resolution data in nearby galaxies becomes available. Using current data, the first striking point is that the ratios mostly exhibit the same order of magnitude independent of scale. This suggests that the emission of the different lines is likely co-spatial to the first order (i.e., the lines arise from the same molecular gas phase). They are thus affected by beam dilution in similar ways. Detailed analysis of second-order variations of the ratios may indicate other physical and chemical processes.

In extragalactic work, the $\mathrm{HCN}$-to-CO ratio is often used as an indicator of gas density. The ratio measured on $140 \mathrm{pc}$ scale in the headlight cloud, $\mathrm{HCN} / \mathrm{CO}=0.025$ to 0.04 , is in broad agreement with the values found in galaxy disks (e.g., Usero et al. 2015; Gallagher et al. 2018). It is higher than the 
A\&A 634, A121 (2020)

Table 5. ${ }^{13} \mathrm{CO} /{ }^{12} \mathrm{CO}(1-0), \mathrm{CO}(2-1) / \mathrm{CO}(1-0), \mathrm{HCO}^{+} / \mathrm{HCN}$, and $\mathrm{HCN} / \mathrm{CO}(1-0)$ values for the different sources shown in Fig. 6.

\begin{tabular}{|c|c|c|c|c|c|c|c|c|c|c|c|c|c|c|c|c|c|}
\hline \multirow[t]{2}{*}{ Source } & \multicolumn{4}{|c|}{${ }^{12} \mathrm{CO} /{ }^{13} \mathrm{CO}(1-0)$} & \multicolumn{4}{|c|}{$\mathrm{CO}(2-1) / \mathrm{CO}(1-0)$} & \multicolumn{4}{|c|}{$\mathrm{HCO}^{+} / \mathrm{HCN}(1-0)$} & \multicolumn{4}{|c|}{$\mathrm{HCN} / \mathrm{CO}(1-0)$} & \multirow[t]{2}{*}{ Ref. } \\
\hline & Size & & Mean ratio & & Size & & Mean ratio & & Size & & Mean ratio & & Size & & Mean ratio & & \\
\hline Headlight & $140 \mathrm{pc}$ & & 7.20 & & $140 \mathrm{pc}$ & & 0.70 & & $140 \mathrm{pc}$ & & 1.0 & & $140 \mathrm{pc}$ & & 0.03 & & This work \\
\hline Orion B & $6.5 \mathrm{pc}$ & & 6.50 & & $6.5 \mathrm{pc}$ & & 0.75 & & $6.5 \mathrm{pc}$ & & 1.10 & & $6.5 \mathrm{pc}$ & & 0.92 & & S94, P17 \\
\hline 30 Dor- 10 & $8 \mathrm{pc}$ & & 6.47 & & $10 \mathrm{pc}$ & & 1.07 & & $1.5 \mathrm{pc}^{(\dagger)}$ & & 5 & & $1.5 \mathrm{pc}^{(\dagger)}$ & & 0.14 & & $\mathrm{P} 12, \mathrm{~J} 98, \mathrm{~A} 14$ \\
\hline \multicolumn{2}{|c|}{ EMPIRE galaxies } & Gal. & Disk & Cen. & & Gal. & Disk & Cen. & & Gal. & Disk & Cen. & & Gal. & Disk & Cen. & C18, JD \\
\hline NGC 6946 & $0.9 \mathrm{kpc}$ & 13.8 & 13.5 & 15.2 & $1.1 \mathrm{kpc}$ & 0.65 & 0.64 & 0.68 & $1.1 \mathrm{kpc}$ & 1.06 & 1.19 & 0.87 & $1.1 \mathrm{kpc}$ & 0.73 & 0.50 & 1.14 & \\
\hline NGC 5194 & $1.0 \mathrm{kpc}$ & 10.3 & 9.9 & 8.2 & $1.2 \mathrm{kpc}$ & 0.73 & 0.81 & 0.80 & $1.2 \mathrm{kpc}$ & 0.75 & 0.97 & 0.65 & $1.2 \mathrm{kpc}$ & 1.11 & 1.17 & 2.26 & \\
\hline NGC 5055 & $1.0 \mathrm{kpc}$ & 9.3 & 8.5 & 7.2 & $1.3 \mathrm{kpc}$ & 0.59 & 0.59 & 0.68 & $1.3 \mathrm{kpc}$ & 0.33 & 0.35 & 0.90 & $1.3 \mathrm{kpc}$ & 1.19 & 1.07 & 0.71 & \\
\hline NGC 2903 & $1.2 \mathrm{kpc}$ & 10.3 & 11.0 & 12.3 & $1.4 \mathrm{kpc}$ & 0.63 & 0.64 & 0.68 & $1.4 \mathrm{kpc}$ & 0.53 & 0.42 & 0. & $1.4 \mathrm{kpc}$ & 0.92 & 0.83 & 1.38 & \\
\hline NGC 3627 & $1.2 \mathrm{kpc}$ & 12.0 & 12.2 & 15.2 & $1.5 \mathrm{kpc}$ & 0.48 & 0.47 & 0.48 & $1.5 \mathrm{kpc}$ & 0.90 & 0.87 & 0.91 & $1.5 \mathrm{kpc}$ & 0.76 & 0.59 & 0.70 & \\
\hline NGC 0628 & $1.3 \mathrm{kpc}$ & 13.2 & 13.5 & 9.9 & $1.5 \mathrm{kpc}$ & 0.61 & 0.64 & 0.57 & $1.5 \mathrm{kpc}$ & 0.55 & 0.47 & 0.89 & $1.5 \mathrm{kpc}$ & 0.54 & 0.72 & 0.63 & \\
\hline NGC 3184 & $1.5 \mathrm{kpc}$ & 10.2 & 10.7 & 11.2 & $1.9 \mathrm{kpc}$ & 0.53 & 0.53 & 0.52 & $1.9 \mathrm{kpc}$ & 0.52 & 0.55 & 0.77 & $1.9 \mathrm{kpc}$ & 0.42 & 0.39 & 0.62 & \\
\hline NGC 4321 & $1.9 \mathrm{kpc}$ & 10.3 & 10.3 & 10.9 & $2.3 \mathrm{kpc}$ & 0.57 & 0.56 & 0.62 & $2.3 \mathrm{kpc}$ & 0.90 & 1.03 & 0.75 & $2.3 \mathrm{kpc}$ & 1.14 & 0.84 & 1.57 & \\
\hline NGC 4254 & $1.9 \mathrm{kpc}$ & 11.0 & 10.5 & 8.4 & $2.3 \mathrm{kpc}$ & 0.74 & 0.74 & 0.75 & $2.3 \mathrm{kpc}$ & 1.19 & 1.23 & 0.79 & $2.3 \mathrm{kpc}$ & 0.75 & 0.70 & 1.23 & \\
\hline
\end{tabular}

Notes. For nearby galaxies from the EMPIRE survey, the average ratios are given for entire galaxies, disks, and centers. The physical sizes used to derive these ratios are also listed. ${ }^{(\dagger)}$ The lines were observed at an angular resolution corresponding to $29 \mathrm{pc}$.

References. S94: Sakamoto et al. (1994); P17: Pety et al. (2017); P12: Pineda et al. (2012); J98: Johansson et al. (1998); A14: Anderson et al. (2014); C18: Cormier et al. (2018); JD: Jiménez-Donaire et al. (2019).

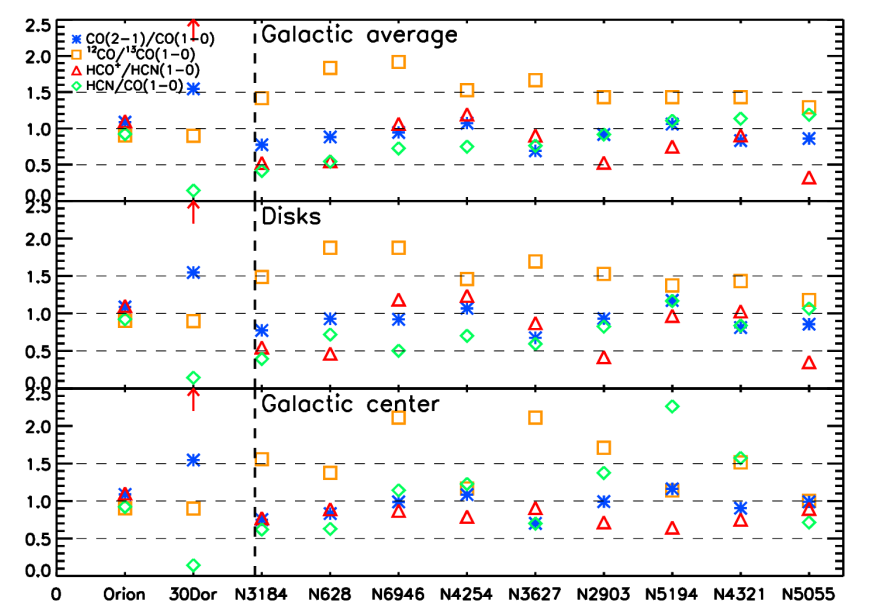

Fig. 6. Line ratios observed in different sources, normalized to the values measured for the headlight cloud in NGC 628. Yellow, blue, red, and green symbols correspond to the ${ }^{12} \mathrm{CO} /{ }^{13} \mathrm{CO}, \mathrm{CO}(2-1) / \mathrm{CO}(1-0)$, $\mathrm{HCO}^{+} / \mathrm{HCN}$, and $\mathrm{HCN} / \mathrm{CO}(1-0)$ values. The values given for 30 Dor correspond to the values in the 30 Dor- 10 molecular cloud. The red arrow for 30 Dor indicates that the value of $\mathrm{HCO}^{+} / \mathrm{HCN}$ is higher than 2.5. See Table 5 for the absolute values for these line ratios and their references.

value measured on larger scales from the EMPIRE full-disk map of NGC 628 (Jiménez-Donaire et al. 2019), for which the mean value of $\mathrm{HCN} / \mathrm{CO}$ is $\sim 0.015$. This can be seen from the low normalized value, $\sim 0.5$, of the green symbol in NGC 628 in Fig. 6, showing that the value of $\mathrm{HCN} / \mathrm{CO}$ in the headlight cloud is about two times higher than the galaxy-averaged value. Assuming that $\mathrm{HCN} / \mathrm{CO}$ highlights dense gas, the headlight cloud appears to be denser than its surroundings, with more dense $\mathrm{HCN}$-emitting gas, consistent with the compact structure at the core of the cloud structure measured in $\mathrm{CO}$.

The value of $\mathrm{HCO}^{+} / \mathrm{HCN}$ is more variable among the EMPIRE survey targets. The value of $\mathrm{HCO}^{+} / \mathrm{HCN}$ in the headlight cloud is about twice the disk-averaged value within NGC 628 (0.55), and closer to the value estimated toward its central region (0.89). It is similar to the average ratio observed in Orion B (1.1), but significantly lower than the ratio observed in the 30 Dor-10 molecular cloud (5). We note that $\mathrm{HCO}^{+} / \mathrm{HCN}$ values ranging between 3 and 10 have been reported for several star-forming regions in the LMC (including N159W, N44, N105, and other clouds near 30 Dor, Seale et al. 2012; Anderson et al. 2014) and the low-metallicity Local Group dwarf IC 10 (Nishimura et al. 2016; Braine et al. 2017; Kepley et al. 2018). This may indicate a metallicity dependence of this ratio, since $\mathrm{HCO}^{+} / \mathrm{HCN}$ values close to unity are observed in massive star-forming regions in our Galaxy (e.g., W51 Watanabe et al. 2017), and values between $0.5-2$ on larger scales in starburst galaxies (e.g., Imanishi et al. 2007; Krips et al. 2010; Bemis \& Wilson 2019).

The ${ }^{12} \mathrm{CO} /{ }^{13} \mathrm{CO}$ value in the headlight cloud is 7.2 , which is comparable to the value in Orion B and in the 30 Dor- 10 molecular cloud. The EMPIRE measurements for NGC 628 and other nearby spiral galaxies are systematically higher, which we attribute to the lower filling factor of ${ }^{13} \mathrm{CO}$ emission within the EMPIRE resolution element $\left(27^{\prime \prime} \sim 1\right.$ to $\left.2 \mathrm{kpc}\right)$.

The value of $\mathrm{CO}(2-1) / \mathrm{CO}(1-0)$ in the headlight cloud is $\sim 0.7$, which is close to the standard value for resolved observations in the Milky Way (including the Orion B cloud; see, e.g., Sakamoto et al. 1994, 1997; Yoda et al. 2010), and in nearby galaxies (e.g., Eckart et al. 1990; Leroy et al. 2009, 2013; Cormier et al. 2018). The 30 Dor-10 molecular cloud in the LMC (Pineda et al. 2012) stands out as having a value of $\mathrm{CO}(2-$ $1) / C O(1-0)$ close to unity, while the mean ratio for the kiloparsec scale EMPIRE measurements in NGC 628 and other nearby spiral galaxies is slightly lower, $\sim 0.6$, than in the headlight cloud. The EMPIRE CO $(2-1) / \mathrm{CO}(1-0)$ measurements show some variation between and within galaxies: in NGC 5055 and NGC 4321 the value of $\mathrm{CO}(2-1) / \mathrm{CO}(1-0)$ increases toward the galaxy centers, but the opposite trend is seen for NGC 628.

Since the kiloparsec scale of the EMPIRE measurements can obscure cloud-scale variations of $\mathrm{CO}(2-1) / \mathrm{CO}(1-0)$ within NGC 628, we measured the mean value of $\mathrm{CO}(2-1) / \mathrm{CO}(1-0)$ within a 3 !' $2 \times 3$ '! 2 box, centered on the positions of the GMCs identified in NGC 628 (see Sect. 3.2). To be consistent with the measurements of the headlight cloud in Sect. 3.3.1, the line ratios were defined using the peak brightness of $\mathrm{CO}(1-0)$ and $\mathrm{CO}(2-1)$ data cubes that had been smoothed to common (round beam) resolution of 3 '.2. We restricted our measurement to pixels where the signal-to-noise ratio at the line peak was greater 


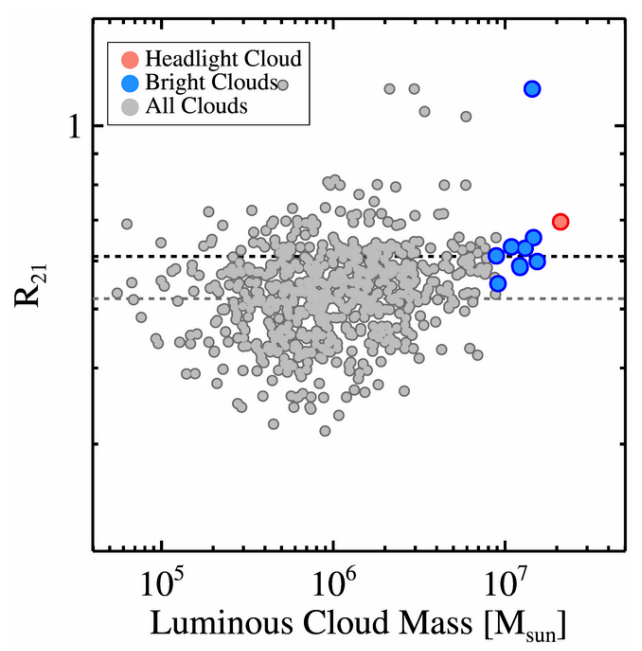

Fig. 7. $\mathrm{CO}(2-1) / \mathrm{CO}(1-0)$ measured on $140 \mathrm{pc}$ scale at the positions of clouds in NGC 628. As in Fig. 3, the headlight cloud is the red point, and the nine next most luminous molecular clouds $\left(M_{\mathrm{lum}}>8.6 \times 10^{6} M_{\odot}\right)$ are in blue. The kiloparsec scale estimate for $\mathrm{CO}(2-1) / \mathrm{CO}(1-0)$ in NGC 628 measured by EMPIRE is indicated with a dashed black line, and the average value for the cloud-scale measurements is represented by a dashed gray line. The $\mathrm{CO}(2-1) / \mathrm{CO}(1-0)$ measurements in this plot use the peak brightness of the $\mathrm{CO}(1-0)$ and $\mathrm{CO}(2-1)$ lines within a 3 ". $2 \times 3$ '. 2 box centered on the cloud position.

than 5 in both tracers, and we rejected clouds where our analysis box contained fewer than ten valid pixels. We list the cloudscale $\mathrm{CO}(2-1) / \mathrm{CO}(1-0)$ measurements for NGC 628's ten most luminous clouds in Table 2, and we plot all the cloud-scale measurements of $\mathrm{CO}(2-1) / \mathrm{CO}(1-0)$ versus the cloud luminous mass in Fig. 7. From this analysis it is clear that kiloparsec scale averages hide genuine local variations in $\mathrm{CO}(2-1) / \mathrm{CO}(1-0)$ : we find a mean cloud-scale value of $\mathrm{CO}(2-1) / \mathrm{CO}(1-0)$ in NGC 628 of 0.54 , with a standard deviation of 0.10 in the cloud-scale measurements. We note here that the headlight cloud has a relatively high $\mathrm{CO}(2-1) / \mathrm{CO}(1-0)$ value relative to the rest of the cloud population of NGC 628, but defer a detailed investigation of the physical origin of $\mathrm{CO}(2-1) / \mathrm{CO}(1-0)$ variations on subkiloparsec scales to a future paper (Saito et al., in prep.).

\subsubsection{LVG modeling}

Here we estimate the typical density and kinetic temperature of the molecular gas of the headlight cloud using a largevelocity gradient (LVG) analysis of some of the line ratios measured at the emission peak of the cloud (see Table 4). We use $\mathrm{RADEX}^{3}$, a public LVG radiative transfer code. We assume an expanding sphere geometry, a velocity linewidth of $20 \mathrm{~km} \mathrm{~s}^{-1}$ (which is the mean value of the observed linewidth for all tracers; see Col. 4 of Table 3), and a background temperature of $2.73 \mathrm{~K}$. The $\mathrm{H}_{2}$ column density is set to $N_{\mathrm{H}_{2}}$ to $9 \times 10^{21} \mathrm{~cm}^{-2}$, which is the value obtained assuming a $\mathrm{CO}$ luminous mass of $2 \times 10^{7} M_{\odot}$ and radius of $180 \mathrm{pc}$ (see Sect. 3.2.1). We first compute a grid of LVG models, which covers a kinetic gas temperature range of $T_{\text {kin }}=10-160 \mathrm{~K}$, and molecular gas density range of $n_{\mathrm{H}_{2}}=10^{2}-10^{7} \mathrm{~cm}^{-3}$. We fit ${ }^{12} \mathrm{CO}(1-0) /{ }^{13} \mathrm{CO}(1-0)$ as a good tracer of temperature variations, $\mathrm{HCN}(1-0) /{ }^{13} \mathrm{CO}(1-0)$ as a tracer of gas density variations, and $\mathrm{HCN}(1-0) /{ }^{12} \mathrm{CO}(2-1)$ as a confirmation of the implied temperature to density ratio.

\footnotetext{
3 http://home.strw.leidenuniv.nl/ moldata/radex.html
}

The molecular abundances are fixed to their typical Galactic values, (i.e., $\left[{ }^{12} \mathrm{CO}\right] /\left[\mathrm{H}_{2}\right]=3 \times 10^{-4},\left[{ }^{12} \mathrm{CO}\right] /\left[{ }^{13} \mathrm{CO}\right]=70$, and $[\mathrm{HCN}] /\left[\mathrm{H}_{2}\right]=5 \times 10^{-9}$ Blake et al. 1987; van Dishoeck \& Black 1988).

We do not attempt to fit the $\mathrm{CO}(2-1) / \mathrm{CO}(1-0)$ and $\mathrm{HCO}^{+}(1-0) / \mathrm{HCN}(1-0)$ values as they are difficult to interpret. While the ${ }^{12} \mathrm{CO}$ lines are optically thick, the energy difference between the $J=2-1$ and $1-0$ levels is only $17 \mathrm{~K}$. The latter property makes $\mathrm{CO}(2-1) / \mathrm{CO}(1-0)$ mostly sensitive to cold gas, while the former property favors tracing diffuse gas at typical temperature of $80 \mathrm{~K}$. The value of $\mathrm{HCO}^{+} / \mathrm{HCN}$ is also difficult to interpret because it is sensitive to the relative abundances of these species, which vary with environment by an order of magnitude (Goicoechea et al. 2019).

The right panel of Fig. 5 shows the result of the fit of the different line ratios as a set of color-coded thick lines for the possible solutions and thin lines showing the $95 \%$ confidence interval. These curves intersect at $n_{\mathrm{H}_{2}} \sim 5 \times 10^{4} \mathrm{~cm}^{-3}$ and $T_{\text {kin }} \sim 20 \mathrm{~K}$. This is more than three orders of magnitude greater than the average $\mathrm{H}_{2}$ density estimated from the cloud mass and diameter quoted above. Assuming that $10 \%$ to $100 \%$ of the mass of the headlight cloud has this typical density and that the geometry is spherical, this yields a typical radius ranging from 15 to $34 \mathrm{pc}$. This gas has a typical thermal pressure of $10^{6} \mathrm{~K} \mathrm{~cm}^{-3}$.

While this solution provides a good fit to the different line ratios, the RADEX-predicted $\mathrm{CO}(2-1)$ brightness is about three times higher than the measured value. We note this as a caveat to the inferred physical conditions, and consider our results for the cloud density and pressure as order-of-magnitude estimates. In view of the uncertainties in the beam filling factor of the different emission lines, the appropriate $X_{\mathrm{CO}}$ value, the molecule abundances, and the density distribution adopted in the LVG model, it is difficult to justify more sophisticated modeling with the current set of molecular line data.

\section{Current stellar feedback}

In this section we characterize the H II region associated with the headlight cloud. In particular, we estimate the typical age and mass of the associated young stellar population and we compute order-of-magnitude pressures related to stellar feedback. More detailed modeling is beyond the scope of this paper. It will be the subject of a forthcoming work.

\subsection{The headlight cloud's H II region}

The headlight cloud spatially coincides with the most luminous H II region within the footprint of our MUSE observations. The $\mathrm{H} \alpha$ emission from this region closely resembles the $\mathrm{CO}$ emission in both morphology and kinematics, suggesting that the H II region is still embedded within the cloud. To within an accuracy of $1^{\prime \prime}$ (i.e., $47 \mathrm{pc}$ ), the two distributions peak at the same position. Figure 8 shows that the velocity structure of the molecular and ionized gas is also very similar.

As in $\mathrm{CO}$, the $\mathrm{H} \alpha$ emission associated with the headlight cloud is the brightest region in the galaxy. This region also appears bright relative to any simple extrapolation of the distribution of intensities from fainter regions. We show this in Fig. 9, where we plot pixel-wise CDFs of the $\mathrm{H} \alpha$ intensity $I_{\mathrm{H} \alpha}$ and $W_{\mathrm{CO}}$ for the entire galaxy.

Figure 9 shows that, like the $\mathrm{CO}$ emission, the $\mathrm{H} \alpha$ emission has a bump at high intensities. The sense of this bump is that there are more bright pixels than expected based on extrapolating from lower intensities. The bump is specifically associated 


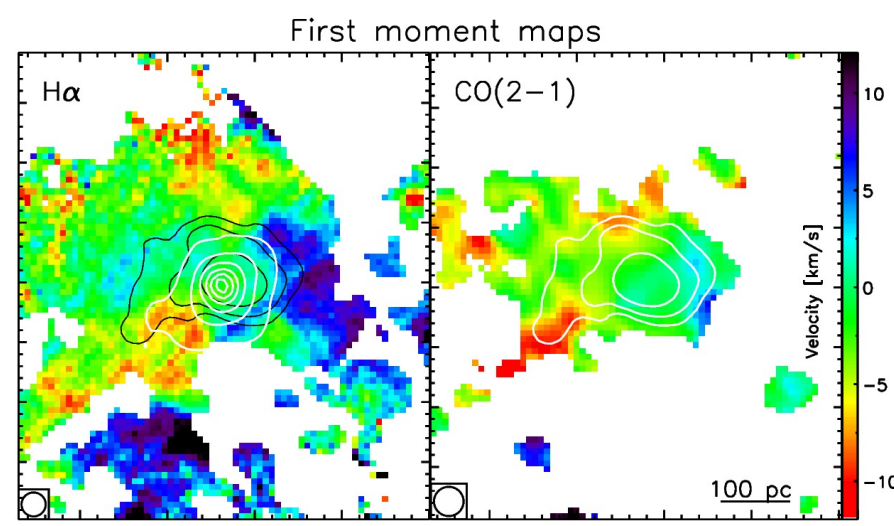

Fig. 8. Maps of the velocity centroid of the $\mathrm{H} \alpha$ and $\mathrm{CO}(2-1)$ emission for the region surrounding the headlight cloud. The maps are constructed after subtracting a model for the galaxy's rotation (Lang et al. 2019). The contours correspond to the $\mathrm{H} \alpha$ and $\mathrm{CO}$ integrated emission: black (white) contours in the left (right) panel are the $\mathrm{CO}(2-1)$ contours, white contours in the left panel are $\mathrm{H} \alpha$ contours. The $\mathrm{H} \alpha$ seeing and the restored $\mathrm{CO}(2-1)$ beam are indicated in the bottom left part of each panel.

with the 45 brightest pixels in the $\mathrm{H} \alpha$ map, which we mark with a green line in Fig. 9. These pixels are all associated with the headlight cloud. They belong to a region of 2.3 square arcseconds in area that spatially correlates with the brightest $\mathrm{CO}$ peak.

\subsection{Young stellar content}

The extinction-corrected $\mathrm{H} \alpha$ luminosity associated with the headlight cloud (Kreckel et al. 2016, 2018) implies a SFR of $0.034 M_{\odot} \mathrm{yr}^{-1}$ assuming a constant star formation history. This is likely a lower limit since the bulk of the star formation activity may still be embedded. Moreover, the star formation history in the headlight cloud region may be better described as an instantaneous burst (see below).

We used the above SFR estimate to assess the local molecular gas depletion time $\tau_{\text {dep }}^{\text {mol }} \equiv M_{\text {mol }} /$ SFR, finding $\tau_{\text {dep }}^{\text {mol }} \sim$ $0.6 \mathrm{Gyr}$ using $2.0 \times 10^{7} M_{\odot}$ for the mass of the cloud. For comparison, we estimated $\tau_{\text {dep }}^{\mathrm{mol}} \sim 1.6 \mathrm{Gyr}$ for the $1 \mathrm{kpc}$ region around the headlight cloud and $\sim 1.5 \mathrm{Gyr}$ for the whole area mapped by ALMA (both consistent with previous work by Leroy et al. 2008, 2013; Bigiel et al. 2008; Kreckel et al. 2018). The latter values represent a large-scale equilibrium rate, while the former smaller value probably represents a small spatialscale snapshot that reflects the current evolutionary state of the headlight (see Schruba et al. 2010; Kruijssen \& Longmore 2014; Kruijssen et al. 2018).

We can obtain a sharper picture of the recent star formation embedded within the headlight cloud by comparing observed properties to Starburst99 (S99) models assuming an instantaneous burst (Leitherer et al. 1999). In this case our best constraint on the age comes from the equivalent width (EW) of the $\mathrm{H} \alpha$ line, and our best constraint on the mass comes from the number of ionizing photons produced, as indicated by the total $\mathrm{H} \alpha$ emission. The observations were compared to models using a solar metallicity, an instantaneous burst of star formation of $10^{6} M_{\odot}$, and a Kroupa initial mass function (IMF).

The age is estimated to be $4 \mathrm{Myr}$ from the measured $\mathrm{H} \alpha \mathrm{EW}$ of $517 \AA$. This is an upper limit since S99 does not yield accurate age measurements when the region is younger than $4 \mathrm{Myr}$. The MUSE spectrum of this H II region shows the presence of

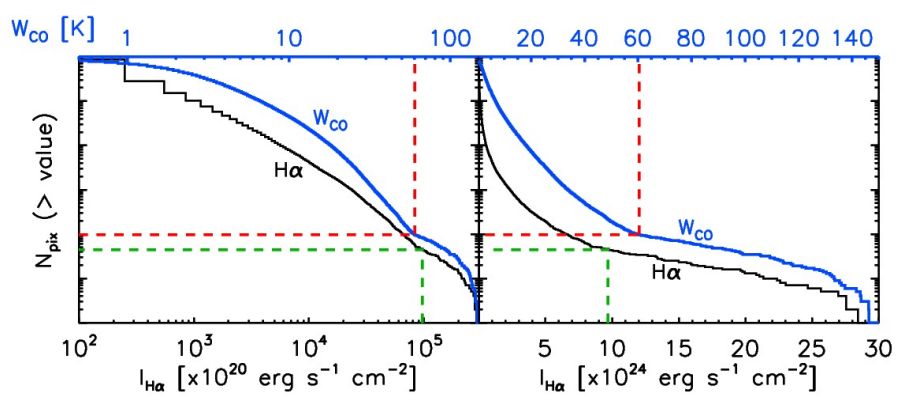

Fig. 9. Number of pixels whose integrated $\mathrm{H} \alpha$ intensity $I_{\mathrm{H} \alpha}$ (black curve) and integrated $\mathrm{CO}(2-1)$ intensity $W_{\mathrm{CO}}$ (blue curve) are above the value given in the bottom abscissa for $I_{\mathrm{H} \alpha}$ and in the top abscissa for $W_{\mathrm{CO}}$, for the entire galaxy. Left panel: full range of values in logarithmic scale. Right panel: zoomed-in linear scale. The red dashed lines are as in Fig. 1. The green dashed lines show the values corresponding to 45 pixels (or a surface of about $11^{\prime \prime} 5 \times 11^{\prime \prime} 5$ ) in the $\mathrm{H} \alpha$ map.

the C IV line at $15801-12 \AA$. This line is a specific feature of Wolf-Rayet stars, which are present in stellar populations aged between 2 and $6 \mathrm{Myr}$. Combining the two results, the age of the young stellar population is constrained to $2-4 \mathrm{Myr}$.

The mass in young stars is estimated from the number of ionizing photons, $N_{\text {ion }}$, they produce, which itself is estimated from the integrated extinction-corrected $\mathrm{H} \alpha$ luminosity of the headlight cloud's H II region $L(\mathrm{H} \alpha)=6.3 \times 10^{39} \mathrm{erg} \mathrm{s}^{-1}$. Using Eq. (5) from Calzetti et al. (2010), this luminosity yields $N_{\text {ion }}=$ $4.6 \times 10^{51}$ photon $^{-1}$. The $\mathrm{S} 99$ single stellar population models assuming this value of $N_{\text {ion }}$ yield a stellar mass as high as $M_{\mathrm{cl}} \sim$ $3 \times 10^{5} M_{\odot}$ and a bolometric luminosity of $L_{\mathrm{cl}}=4 \times 10^{8} L_{\odot}$. The measured and estimated properties of the young massive stellar population embedded in the headlight cloud are summarized in Table 6.

\subsection{Stellar feedback}

The spatial coincidence of a massive young stellar population and massive GMC indicates that these stars have not yet dispersed their parent molecular cloud. In this section we investigate the potential feedback mechanisms that may lead to the disruption of the headlight cloud, adopting the properties for the cloud and the young stellar population listed in Tables 2 and 6 , respectively.

The population of Wolf-Rayet stars produces strong winds that will push away the low-density ionized gas of the H II region. In the simple spherically symmetric picture, this results in a thin or thick shell of ionized gas surrounding an almost empty central region. The density in the shell will be higher than the mean density of the sphere, so that the ram pressure approximately balances the thermal pressure.

To examine the balance of forces in more detail, we start by assuming that the hot gas associated with the wind has been lost from the young stars, either because it has already radiatively cooled or because it has escaped through low-density gaps in the shell (see, e.g., Rogers \& Pittard 2013). This assumption is consistent with the fact that we do not find any signature of compact X-ray emission associated with the headlight cloud in Chandra and XMM-Newton observations (Liu 2005; Owen \& Warwick 2009). It is also consistent with detailed models of the effects of stellar winds on molecular clouds, which typically find that the hot gas is lost within only $\sim 1$ Myr from the onset of the wind (see, e.g., Rahner et al. 2017). The mechanical luminosity of the wind predicted by the $S 99$ models is $L_{\text {mech }}=4.9 \times 10^{6} L_{\odot}$. This 
Table 6. Estimated physical properties of the H II region and young stellar population embedded in the headlight cloud in NGC 628.

\begin{tabular}{lcc}
\hline \hline Name & Symbol & Value \\
\hline $\mathrm{H} \alpha$ luminosity & $L_{\mathrm{H} \alpha}$ & $6.3 \times 10^{39} \mathrm{erg} \mathrm{s}^{-1}$ \\
$\mathrm{H} \alpha$ region radius & - & $142 \mathrm{pc}$ \\
$\mathrm{H} \alpha$ linewidth & $\Delta v$ & $\sim 50 \mathrm{~km} \mathrm{~s}^{-1}$ \\
$\mathrm{H} \alpha$ equivalent width & $W_{\mathrm{H} \alpha}$ & $517 \AA$ \\
Ionizing photon production rate & $N_{\mathrm{ion}}$ & $4.6 \times 10^{51} \mathrm{phot.}^{-1}$ \\
Young star mass & $M_{\mathrm{cl}}$ & $3 \times 10^{5} M_{\odot}$ \\
Young star bolometric luminosity & $L_{\mathrm{cl}}$ & $4 \times 10^{8} L_{\odot}$ \\
Young star typical age & - & $2-4 \mathrm{Myr}$ \\
\hline
\end{tabular}

can also be written in terms of the total stellar mass loss rate $(\dot{M})$ through

$L_{\text {mech }}=\frac{1}{2} v_{\text {wind }}^{2} \dot{M}$,

where $v_{\text {wind }}$ is the characteristic wind velocity. In the present case the wind luminosity is dominated by the Wolf-Rayet stars, and thus the wind velocity will typically be very high (i.e., $v_{\text {wind }} \sim$ $2 \times 10^{3} \mathrm{~km} \mathrm{~s}^{-1}$ ). This yields a mass loss rate $\ddot{M} \sim 9.4 \times 10^{23} \mathrm{~g} \mathrm{~s}^{-1}$. The ram pressure at radius $R$ is

$P_{\text {ram,wind }}=\frac{v_{\text {wind }} \dot{M}}{4 \pi R^{2}}$.

Assuming that $R$ is the radius of the H II region, we find $P_{\text {ram,wind }} \sim 5.7 \times 10^{5} \mathrm{~K} \mathrm{~cm}^{-3}$. This value depends on our assumed wind velocity, since $P_{\text {ram,wind }} \propto v_{\text {wind }}^{-1}$, but does not change by more than a factor of two for wind velocities in the range consistent with Wolf-Rayet stars.

If some of the massive young stars have already exploded as supernovae ( $\mathrm{SNe}$ ), they also contribute to the total ram pressure. The importance of this contribution depends on the age of the young stellar population and the assumed star formation history. For our assumption of an instantaneous burst, the first supernovae occur at a time $t \sim 3.6 \mathrm{Myr}$ (Leitherer et al. 1999). The headlight cloud appears as a compact source in the nonthermal emission map at $3.1 \mathrm{GHz}$ in Mulcahy et al. (2017), thus likely tracing synchrotron emission from supernovae. We can estimate the contribution that the associated supernovae make to the ram pressure from

$P_{\mathrm{ram}, \mathrm{SN}}=\frac{v_{\mathrm{SN}} \dot{M}_{\mathrm{SN}}}{4 \pi R^{2}}$

Here $\dot{M}_{\mathrm{SN}}$ is the supernova mass loss rate, which we take from the $\mathrm{S} 99$ model, and $v_{\mathrm{SN}}$ is the terminal velocity of the supernova ejecta, which we take to be $10^{4} \mathrm{~km} \mathrm{~s}^{-1}$, following Rahner et al. (2017). We note that this estimate is conservative, in that it neglects any contribution made by the pressure of the hot gas associated with the supernovae, consistent with our treatment of the stellar winds. For a young stellar population age of $4 \mathrm{Myr}$, this yields a ram pressure contribution $P_{\text {ram,SN }} \sim 3.0 \times 10^{5} \mathrm{~K} \mathrm{~cm}^{-3}$ (i.e., roughly half of the contribution coming from stellar winds). We recover similar results for other assumed ages in the range $3.6<t<6.0 \mathrm{Myr}$.

At the radius of the $\mathrm{H}$ II region, the wind ram pressure balances the thermal pressure due to the ionized photons (Pellegrini et al. 2007), provided that other sources of support (e.g., magnetic fields) are unimportant. If we assume that thermal pressure is the main source of support for the ionized shell, and that the gas has a typical $\mathrm{H}$ II region temperature of $10^{4} \mathrm{~K}$, then it follows that the ionized gas must have a typical electron density of $n_{\mathrm{e}} \sim 30 \mathrm{~cm}^{-3}$. If we assume that this density is achieved in a shell with radius $R_{\mathrm{H} \text { II }}$, we can also solve for the shell thickness $(\mathrm{d} R)$ required to have ionization balance

$N_{\text {ion }}=\frac{4 \pi}{3} \alpha_{B} n_{\mathrm{e}} R_{\mathrm{H} \text { II }}^{2} \mathrm{~d} R$,

where $\alpha_{B}$ is the case $\mathrm{B}$ recombination coefficient. This gives $\mathrm{d} R \sim 7 \mathrm{pc}$. We note that this is technically the thickness of the ionized portion of the shell: if the expansion of the shell has also swept up a substantial amount of neutral and/or molecular gas, then the full shell will be even thicker. Finally, we can calculate the mass of the ionized gas: $M_{\text {ion }} \sim 6 \times 10^{5} M_{\odot}$. The ionized gas thus does not contribute much to the overall mass budget. For comparison, Galván-Madrid et al. (2013) find that in W49 (one of the most massive GMCs in the Milky Way, albeit a factor of 10 less massive than the headlight cloud) only $\sim 1 \%$ of the total gas mass is in the ionized component, comparable to the value of a few percent we infer here for the headlight cloud.

The radiation pressure is estimated following Eq. (1) in Herrera \& Boulanger (2017). For a radius defined by the $\mathrm{H} \alpha$ emission, and assuming no trapping of the IR photons within the shell, the radiation pressure is $P_{\text {rad }}=1.5 \times 10^{5} \mathrm{~K} \mathrm{~cm}^{-3}$. The turbulent pressure in the molecular gas, $P_{\text {turb }}=\rho \sigma_{v}^{2}$, is estimated to be $P_{\text {turb }}=1.9 \times 10^{5} \mathrm{~K} \mathrm{~cm}^{-3}$.

The magnetic pressure cannot be accurately computed, as we have no measurement of the magnetic field strength on the scale of the headlight cloud. If we adopt a value of $10 \mu \mathrm{G}$, based on the kiloparsec scale measurements of Mulcahy et al. (2017), the resulting magnetic pressure is $P_{\text {mag }}=2.9 \times 10^{4} \mathrm{~K} \mathrm{~cm}^{-3}$, implying that the field would not play a significant role in regulating the expansion of the H II region (van Marle et al. 2015). It is possible that the cloud-scale magnetic field strength is greater than this, implying a higher magnetic pressure, but we do not expect $P_{\text {mag }}$ to exceed the turbulent pressure unless the turbulence in the cloud is substantially sub-Alfvénic. Since it would be difficult to explain the large amounts of recent star formation if the cloud were strongly magnetically dominated, we conclude that $P_{\text {mag }}$ is unlikely to greatly exceed $P_{\text {turb }}$.

The gravitational pressure exerted by the young stellar body and the molecular cloud itself is $P_{\text {grav }}=3.6 \times 10^{5} \mathrm{~K} \mathrm{~cm}^{-3}$, following Eq. (5) in Herrera \& Boulanger (2017), assuming a size defined by the $\mathrm{CO}$ emission. In this computation, we neglected the contribution of the ionized component, as discussed above. Hence, the combined outward pressures, $P_{\text {turb }}, P_{\text {ram }}$ (winds and supernovae), and $P_{\text {rad }}$, overcome the gravitational and magnetic pressures by a factor of $2-4$.

\subsection{Comparison with 30 Dor in the LMC}

We note that the properties of the H II region in the headlight cloud are similar to those of N157A (Henize 1956), the H II region associated with the 30 Dor star-forming region, which is the largest and most active star-forming region in the Local Group. N157A has an ionizing photon rate of $N_{\text {ion }}=4.5 \times$ $10^{51}$ photon $^{-1}$ and a size of $\sim 200 \mathrm{pc}$ (Walborn 1991); the H II region in the headlight cloud has $N_{\text {ion }}=4.6 \times 10^{51}$ photon s$^{-1}$ and a radius of $\sim 140 \mathrm{pc}$ (see Table 6 ). The age and mass of the embedded young stars in the headlight cloud are likewise comparable to the 30 Dor central star cluster (R136), which is $\sim 1-3$ Myr old and has a stellar mass of $4.5 \times 10^{5} M_{\odot}$ (Bosch et al. 2009). The molecular gas properties of these regions are quite 

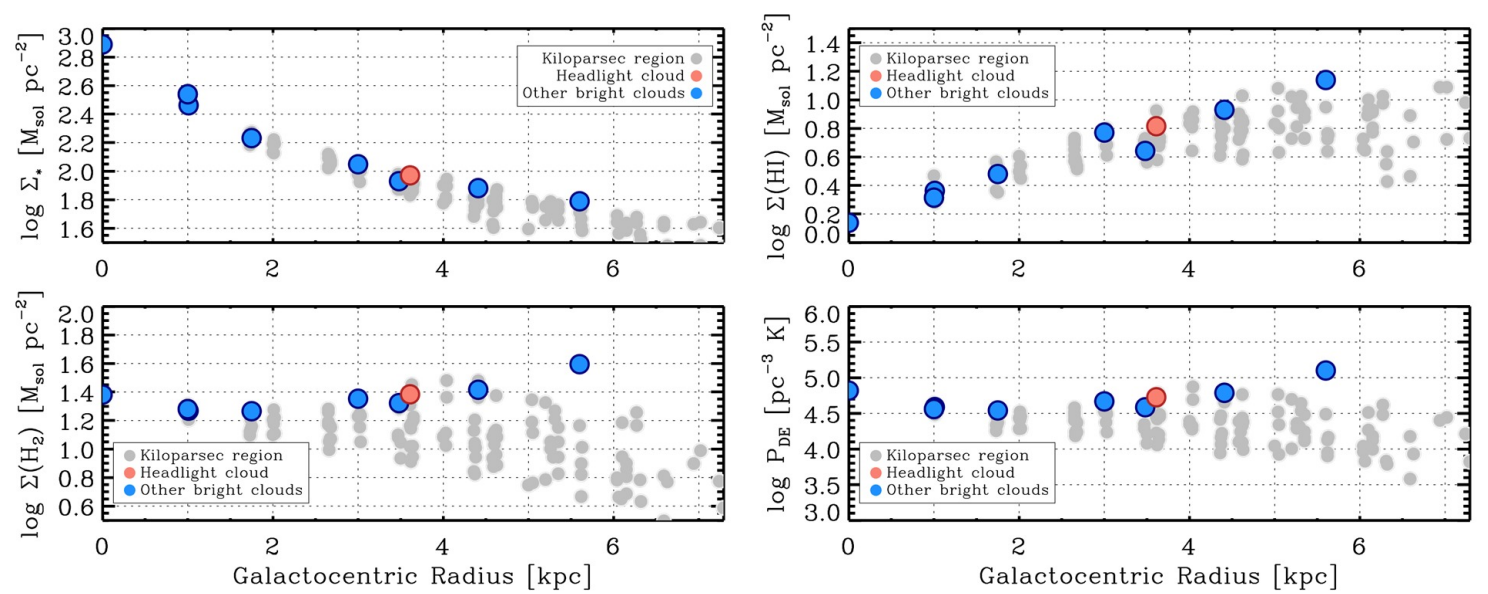

Fig. 10. Headlight cloud in galactic context. Panels display the average surface density of stellar mass (top left), atomic gas (top right), molecular gas (bottom left), and estimated dynamical equilibrium pressure (bottom right) as a function of the galactocentric radius in kiloparsec-sized apertures that cover the galaxy (from Sun et al., in prep.). The gray points show all apertures, and the colored points show apertures that host one of the ten brightest clouds in the galaxy (red: headlight cloud, blue: another bright cloud). The vertical spread of points at a fixed radius indicates azimuthal variations.

different, however. The integrated ${ }^{12} \mathrm{CO}(1-0)$ luminosity that is observed to be spatially projected onto N157A is $\sim 2.5 \times$ $10^{5} \mathrm{~K} \mathrm{~km} \mathrm{~s}^{-1} \mathrm{pc}^{2}$, corresponding to only $\sim 10^{6} M_{\odot}$ of molecular gas for a Galactic CO-to- $\mathrm{H}_{2}$ conversion factor (Wong et al. 2011; Johansson et al. 1998). The young stars in 30 Dor thus appear to have cleared out most of the molecular gas associated with the H II region, contrary to the headlight cloud, for which we estimate a molecular mass of $\geq 10^{7} M_{\odot}$.

\section{Galactic environment of the headlight cloud}

Both the properties and location of the headlight cloud make it remarkable, as massive star-forming regions usually tend to appear at bar ends or galaxy centers. In this section we examine the environment of the headlight cloud. First, we compare the properties of the region around the cloud to the rest of the galaxy. Then we consider how the cloud's location relates to the dynamical structure of the galaxy.

\subsection{Trends with galactocentric radius}

In Fig. 10, we show the kiloparsec scale structure of NGC 628, highlighting the location of the headlight cloud. We plot the gas and stellar structure in NGC 628 as a function of galactocentric radius. Each point in Fig. 10 shows mean properties of NGC 628 estimated within a $1 \mathrm{kpc}$ aperture (for details, see Leroy et al. 2016; Sun et al. 2018; Utomo et al. 2018). The apertures cover the galaxy. The measurements are taken from Sun et al. (in prep.).

The measurements shown in Fig. 10 are derived from multi-wavelength archival data for NGC 628. In addition to the PHANGS-ALMA CO(2-1) maps, we use the THINGS HI $21 \mathrm{~cm}$ moment-0 map (Walter et al. 2008) to trace atomic gas distribution, the $\mathrm{S}^{4} \mathrm{G}$ dust-subtracted $3.6 \mu \mathrm{m}$ image (Sheth et al. 2010; Querejeta et al. 2015) to trace stellar mass distribution, and GALEX-NUV and WISE band-3 images (presented in Leroy et al. 2019) to trace the distribution of star formation activities. We translate these observations into physical units (e.g., $M_{\odot} \mathrm{pc}^{-2}$ ) following standard techniques, as presented in the above-mentioned publications. We plot the stellar surface density, atomic gas surface density, molecular gas surface density, and the estimated dynamical equilibrium pressure, all as a function of radius. We indicate the location of the region con- taining the headlight cloud in red and show the environments containing the next brightest clouds (i.e., the other nine clouds in Table 2) in blue.

The top left panel in Fig. 10 shows stellar surface density as a function of radius. The stellar mass in NGC 628 is dominated by a relatively smooth exponential disk. Here the regions hosting bright clouds appear unremarkable. Many lie far from the galaxy center $(\sim 3-5 \mathrm{kpc})$ and at comparatively low stellar surface density. Both the large radius and low stellar surface density may be surprising. As emphasized above, dense gas and high surface density gas tend to be more common in galaxy centers (see, e.g., Gallagher et al. 2018; Sun et al. 2018, and references therein). Meanwhile, the stellar surface density plays a key role in setting the interstellar medium (ISM) equilibrium pressure (e.g., Elmegreen 1989; Ostriker et al. 2010). ISM pressure has been found to correlate with the prevalence and properties of molecular gas (e.g., Wong \& Blitz 2002; Blitz \& Rosolowsky 2006; Leroy et al. 2008; Hughes et al. 2013). These clouds lie at large radius and low stellar surface density, and so we might expect the gas to be mostly atomic and full of clouds with modest internal pressures.

The top right and bottom left panels show that the atomic gas and molecular gas surface densities in NGC 628 become comparable beyond $\sim 4-5 \mathrm{kpc}$, while the inner part of the galaxy is more molecule rich. While bright clouds are found at these radii, the bottom right panel shows that they tend to be found in environments where the gas is predominantly molecular. Outside the galaxy center, the molecular gas surface density shows large spread at fixed radius, often $\sim 0.5 \mathrm{dex}$ (a factor of three) or more. This reflects that the strong spiral structure dominates the morphology of the galaxy, leading to a high degree of azimuthal structure. The bright clouds all appear among the highest surface density points at their radii. Moreover, the regions with bright clouds at $r_{\text {gal }} \sim 3-4 \mathrm{kpc}$ have molecular gas surface densities comparable to the galaxy center. This was not necessarily the case, making regions with bright clouds remarkable in NGC 628.

The last panel combines the information from the first three panels following Ostriker et al. (2010) and Elmegreen (1989) to estimate the mean dynamical equilibrium pressure. The formulae and detailed calculation is presented in Sun et al. (in prep.), but the version plotted here follows Gallagher et al. (2018) closely (see that paper for more details). Qualitatively, this 
quantity represents the mean pressure needed to support the weight of the ISM due to both self-gravity and stars. As mentioned above, it has been shown to correlate with both molecular content and the properties of molecular clouds.

The bright clouds, including the headlight cloud, all lie at the high end of the pressures found in NGC 628. This is true despite the comparatively low stellar surface density, indicating that selfgravity of the gas plays a large role here. Along with the significant azimuthal scatter also visible in the bottom right panel, this again emphasizes the important role of galactic dynamics in creating these clouds.

Figure 10 paints a picture of a relatively quiescent galaxy in which the location of massive clouds is driven by the spiral structure. NGC 628 lacks a bulge, stellar bar, or other feature to drive nuclear gas concentrations. Its overall surface density of gas and stars is modest. As a result, the concentration of gas by spiral arms creates some of the highest pressure regions in the galaxy, with the pressure driven by gas self-gravity. The bright clouds identified by CPROPS, including the headlight cloud, preferentially fall along these arms.

Given the apparent central role of dynamics in concentrating molecular gas to create these clouds, we turn our attention to this topic in the next section.

\subsection{Relation to the spiral arms and radial inflows}

Here we consider the particular properties and star formation activity of NGC 628's headlight cloud in relation to the large-scale dynamical structure of the disk. Figure 11 shows the spatial distribution of GMCs in the Rosolowsky et al. (in prep.) catalog, using color to represent the CO luminous mass, velocity dispersion, virial parameter, and molecular gas surface density of the clouds. The size of the symbol corresponds to the cloud size. The shaded light yellow circles represent corotation radii of $3.2 \mathrm{kpc}$ (red circles) and $5.1 \mathrm{kpc}$ (green circles) (see text below, Cepa \& Beckman 1990; Fathi et al. 2007), with an uncertainty of $15 \%$.

It is evident from Fig. 11 that, as suggested in the previous section, large massive clouds in NGC 628, including the headlight cloud, are located preferentially in the two prominent, tightly wrapped, spatially extended spiral arms. This suggests that the pattern of gas flow along and through the spiral arms may be important for determining the growth, longevity, and properties of these clouds. The gas response to the spiral pattern has already been studied in detail by Fathi et al. (2007). They applied the Tremaine-Weinberg method to the ionized gas kinematics to measure a pattern speed $\Omega_{\mathrm{p}}$ that is consistent with the corotation radius at $5.1 \mathrm{kpc}$ identified earlier by Cepa \& Beckman (1990). This cororation radius lies near the edge of our map (green circles in Fig. 11).

In the presence of a single spiral pattern, with a single corotation radius, all the clouds within our field of view, including the headlight, would interact with the spiral pattern on a timescale of $\tau_{\mathrm{sp}}=\pi /\left(\Omega-\Omega_{\mathrm{p}}\right)$. This yields $\tau_{\mathrm{sp}}=140 \mathrm{Myr}$ at the location of the headlight cloud. However, from the detailed kinematic analysis of Fathi et al. (2007), we infer a second inner corotation radius at $R \approx 65^{\prime \prime}=3.2 \mathrm{kpc}$, very near the position of the headlight cloud. In this case, the headlight would remain essentially motionless with respect to the spiral pattern over its entire life, as discussed below.

Following Wong et al. (2004), the second corotation can be inferred from the pattern in the coefficients of the harmonic decomposition of the line-of-sight velocity field measured by Fathi et al. (2007). We confirm this inner corotation radius using a new measurement of gravitational torques across NGC 628. We followed the method of Querejeta et al. (2016) and García-Burillo et al. (2005), which requires a map of the 2D stellar mass distribution to infer the underlying disk volume density and gravitational potential $\Phi$, and a map of the gas distribution to provide a record of the time-averaged position of the gas relative to the potential. We used the $3.6 \mu \mathrm{m}$ contaminantcleaned map produced by $\mathrm{S}^{4} \mathrm{G}$ Pipeline 5 (Querejeta et al. 2015) for the former, adopting a global $M / L=0.6$ assuming a Chabrier IMF (Meidt et al. 2014). We used the ALMA map shown in the right panel of Fig. 1 to trace the gas mass distribution. Following García-Burillo et al. (2005), we measured the azimuthally averaged radial torque profile as

$\tau(R)=\frac{\int_{\theta}\left[N(x, y)\left(x F_{y}-y F_{x}\right)\right]}{\int_{\theta} N(x, y)}$,

where $R$ and $x, y$ are the radial and Cartesian coordinates, $N(x, y)$ is the gas column density, and the gravitational forces are computed from the reconstructed potential $\Phi$ with $F_{x, y}=\nabla_{x, y} \Phi$. We then measured the differential mass flow rate as

$\frac{d^{2} M(r)}{\mathrm{d} R \mathrm{~d} t}=\left.\left.\tau(R) \frac{1}{L}\right|_{\theta} 2 \pi R N(x, y)\right|_{\theta}$,

assuming that the azimuthal average of the angular momentum is $L_{\theta}=R V_{\text {rot }}$. The resulting profile of inflow driven by gravitational torques is shown in Fig. $12^{4}$. Overall, the torque experienced by the gas in NGC 628 undergoes several sign changes, including a crossing from negative to positive near $R=65^{\prime \prime}=3.2 \mathrm{kpc}$, which gives the location of our inner corotation radius, $\mathrm{CoR}_{\text {in }}$, and corresponds to the galactocentric radius of the headlight cloud.

The position of the headlight cloud very near the location of the inner corotation radius may have important implications for its longevity and ability to grow to its present mass, and thus the vigorous star formation occurring there. As indicated in the top panel of Fig. 12, gravitational torques and (differential) gas flows are zero at corotation. Thus, compared to other locations along the gaseous spiral arms, corotation is a relatively sheltered environment: a cloud at corotation radius is less susceptible to destructive dynamical forces such as shear, and rotates together with the spiral pattern during its entire evolution (i.e., $\left.t_{\mathrm{sp}} \gg t_{\text {orb }}\right)$. Another factor that may promote the growth of the headlight cloud is that NGC 628's inner corotation radius overlaps with one of the inner ultra-harmonic resonances (UHRs) of the outer spiral pattern $\mathrm{UHR}_{\text {out }}$, as illustrated in the bottom panel Fig. 12. In this case, gas sitting in the outer disk that is torqued by the outer spiral would be driven radially inward from the outer corotation radius, $\mathrm{CoR}_{\text {out }}$, toward the $\mathrm{UHR}_{\text {out }}$, providing a continual supply of gas to this region around $\mathrm{CoR}_{\mathrm{in}}$. Following Querejeta et al. (2016), we use the profile of gravitational torques to measure the radial flow across the disk of NGC 628, which we show in the middle panel of Fig. 12. From the measured $\mathrm{d}^{2} M / \mathrm{d} t \mathrm{~d} R$, we estimate that the net mass flow between the inner and outer corotation radius is indeed radially inward, at a net rate $0.07 \pm 0.04 M_{\odot} \mathrm{yr}^{-1}$. The inflow rate is a

\footnotetext{
4 The largest uncertainty on the value at any given location is the stellar mass-to-light ratio used to convert the $3.6 \mu \mathrm{m}$ contaminant-cleaned map into a stellar mass map. Although spatial variations in $M / L$ can lead to variations in the measured torques and gas inflow rates, they are not expected to greatly alter the pattern of sign changes in the torque, which we use to infer the dynamical structure of the spiral pattern. For a detailed assessment of the measurement uncertainty associated with this method of determining the torque profile, see Querejeta et al. (2016).
} 

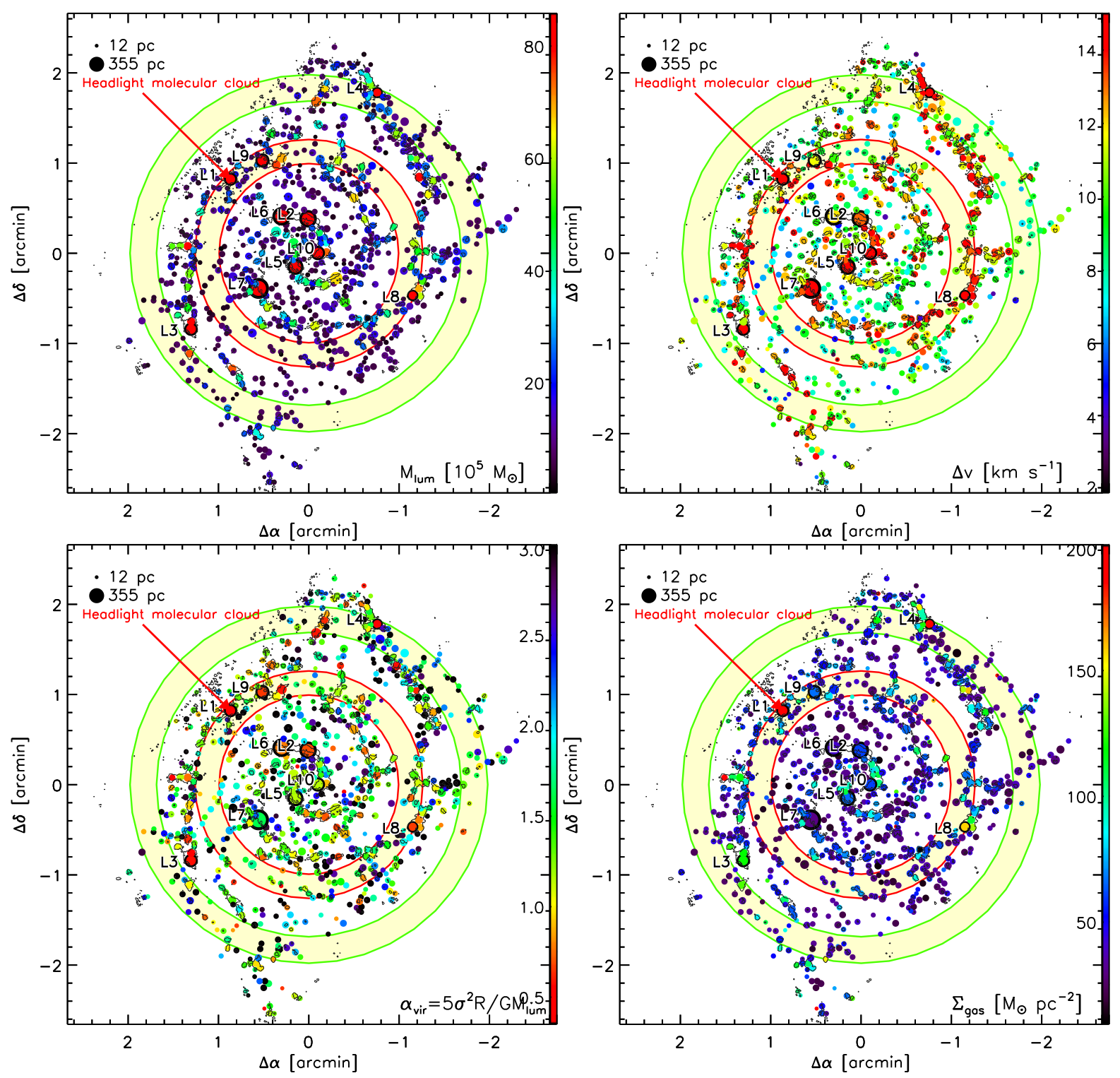

Fig. 11. Position of molecular clouds in NGC 628. From top left to bottom right: colors correspond to the CO luminous masses, CO velocity linewidth, alpha virial parameter, and gas surface density, truncated to $8.6 \times 10^{6} M_{\odot}, 15 \mathrm{~km} \mathrm{~s}^{-1}, 3.0$, and $200 M_{\odot} \mathrm{pc}^{-2}$, respectively. The size of each symbol represents the physical size of the molecular cloud. Indicated in each panel is the headlight molecular cloud (L1) and the nine most massive molecular clouds (L2-L10). The light yellow areas show the position of corotation regions at $3.2 \mathrm{kpc}$ (red circles) and $5.1 \mathrm{kpc}$ (green circles). The contours correspond to the $5 \sigma$ level of the $\mathrm{CO}(2-1)$ moment zero map. Offset positions are from the galactic center.

lower limit since it does not take into account a contribution from viscous torques (which are expected to be dominated by gravity torques; Combes et al. 1990; Barnes \& Hernquist 1996). We develop these ideas as a possible explanation for the mass growth of the headlight cloud in Sect. 6.3.

\section{Discussion}

We have identified the most luminous molecular cloud in NGC 628, the headlight cloud, which is $\sim 2.4$ times brighter in $\mathrm{CO}(2-1)$ than any other molecular cloud in NGC 628. It is the most massive cloud $\left(M_{\text {lum }}=2.0 \times 10^{7} M_{\odot}\right)$ in NGC 628. The cloud is located in a spiral arm at a galactocentric radius of $\sim 3.2 \mathrm{kpc}$, which is coincident with a spiral corotation radius. The cloud itself hosts the most luminous H II region in NGC 628 detected by MUSE, which is associated with a young (2-4 Myr) massive $\left(3 \times 10^{5} M_{\odot}\right)$ stellar population.

\subsection{Overluminous $\mathrm{CO}$ emission in the headlight cloud}

The central $\mathrm{CO}(2-1)$ integrated intensity and peak brightness temperature of the headlight cloud are high compared to the other GMCs in NGC 628, including other massive clouds. While a large integrated intensity could be due to a large cloud linewidth, Fig. 1 shows that the headlight cloud's peak temperature is also about two times greater than other clouds in NGC 628.

More quantitatively, at the $\sim 40 \mathrm{pc}$ resolution of our $\mathrm{CO}(2-1)$ data the average peak brightness temperature of the $\mathrm{CO}(2-1)$ emission from clouds with $M_{\mathrm{vir}} \geq 10^{6} M_{\odot}$ in NGC628 is $T_{\mathrm{mb}}=1.3 \mathrm{~K}$. The headlight cloud peak temperature is $6.7 \mathrm{~K}$. Making a local thermodynamic equilibrium simulation of the radiative transfer, which assumes that the headlight cloud is composed of "standard" molecular gas (i.e., with the characteristic kinetic temperature of $10 \mathrm{~K}$; e.g., Krumholz 2011) filling the full cloud volume, the predicted peak temperature would only be $5.5 \mathrm{~K}$ (i.e., lower than the measured temperature). Alternatively, 


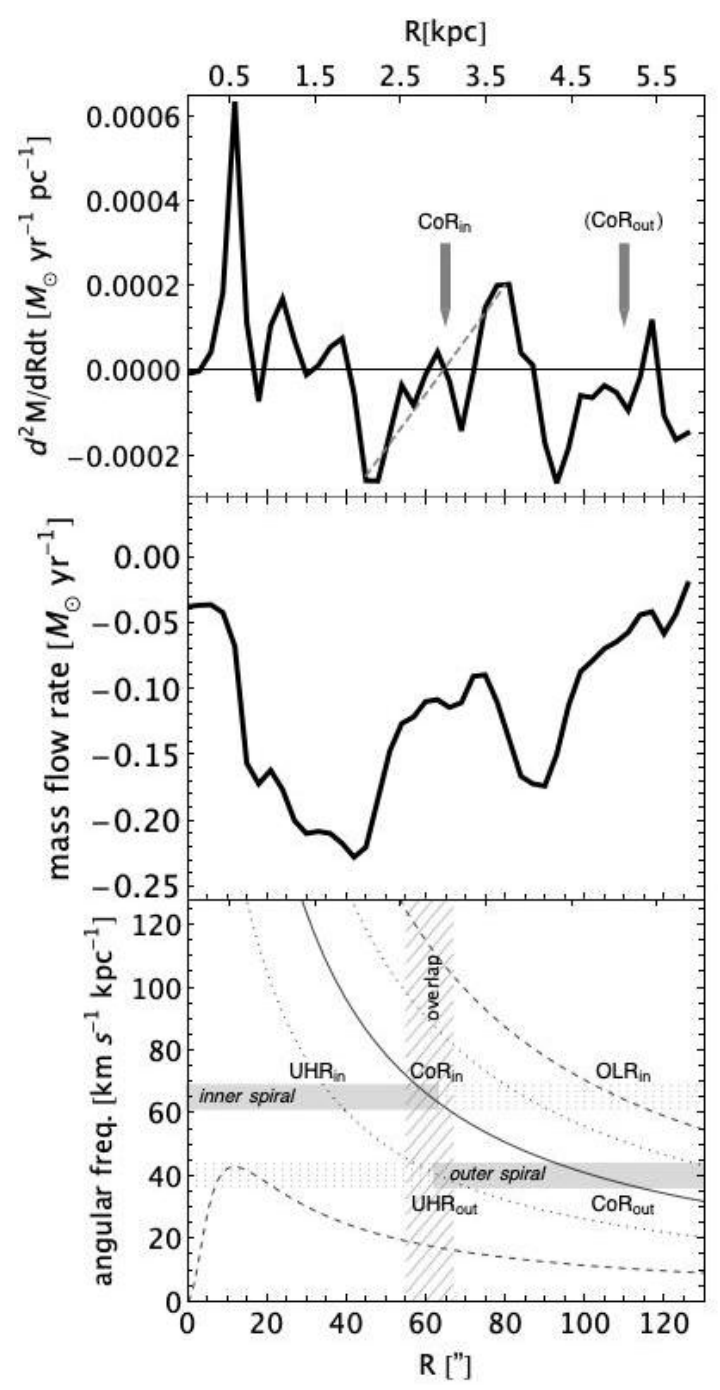

Fig. 12. Top: profile of differential gas flow $\mathrm{d}^{2} M / \mathrm{d} R \mathrm{~d} t$ driven by gravitational torques across NGC 628. The inner corotation radius $\left(\mathrm{CoR}_{\mathrm{in}}\right.$; shown by an arrow) is located where torques and gas flows cross from negative (inward) to positive (outward), as illustrated by the shortdashed segment. The second arrow marks the position of the outer corotation radius, CoR $_{\text {out }}$, previously identified by Cepa \& Beckman (1990), which falls near the edge of the CO field of view. Middle: profile of integrated mass flow rates from the $\mathrm{CoR}_{\text {out }}$ inward to radius $R$. Net flows are radially inward (negative) at all radii. The uncertainty on the inflow (not shown) is dominated by the large, $15^{\prime \prime}$ uncertainty in the position of the $\mathrm{CoR}_{\text {out }}$ near the map edge. Bottom: angular frequency curves in NGC 628, $\Omega$ (solid), $\Omega \pm \kappa / 2$ (dashed) and $\Omega \pm \kappa / 4$ (dotted). Positions of dynamical resonances and corotation radii are indicated. The hashed vertical bar highlights the overlap between the $\mathrm{CoR}_{\text {in }}$ and the outer ultraharmonic resonance.

a typical kinetic temperature of $50 \mathrm{~K}$ would be required so that the headlight cloud has the same beam filling factor of a typical GMC of $10^{6} M_{\odot}$ in NGC 628. Hence, although our measurements do not exclude that there may be an additional increase in the filling factor of the $\mathrm{CO}(2-1)$ emission in the headlight cloud region, we favor an increase of the excitation temperature above $10 \mathrm{~K}$ to explain the headlight cloud's peak brightness because of the spatial coincidence with the HII region heating source.

Another clue that heating is important comes from the $\mathrm{CO}(2-1) / \mathrm{CO}(1-0)$ value, which we measured on $140 \mathrm{pc}$ scales for the cloud population in NGC 628 in Fig. 7. This ratio is $\sim 0.7$ in the headlight cloud, higher than the average ratio for the other bright clouds in Table 2 (0.6), and higher than the average ratio for the overall cloud population (0.54). One possibility is that intense external heating could cause a positive temperature gradient across the photospheres of CO-emitting clumps within the headlight cloud. Due to its higher opacity, the $\mathrm{CO}(2-1)$ transition might then preferentially sample a warmer clump layer than the $\mathrm{CO}(1-0)$ transition, giving a higher value of $\mathrm{CO}(2-1) /$ $\mathrm{CO}(1-0)$. We defer a more detailed investigation of the physical factors linked to variations in this ratio in the disks of PHANGS galaxies to a future paper.

Almost all of the luminous GMCs in NGC 628 listed in Table 2, including the headlight cloud, have low virial parameter values (see Fig. 3) as determined from $\alpha_{\text {vir }}=5 \sigma_{\mathrm{v}}^{2} R / G M_{\text {lum. }}$. If the $\mathrm{CO}(2-1)$ line is overluminous due to excitation effects, then the true mass of the headlight cloud is lower than the luminous mass estimate of $2.0 \times 10^{7} M_{\odot}$. The low virial parameter and bright $\mathrm{CO}(2-1)$ emission in the headlight cloud is thus consistent with the suggestion by Pety et al. (2017) that mass estimates based on the CO luminosity may be biased when GMCs are closely associated with $\mathrm{H} \alpha$ regions, as observed for the Milky Way Orion B cloud. If so, then clouds associated with $\mathrm{H}$ II regions should show lower apparent virial parameters than otherwise identical clouds without nearby heating sources. We intend to test this hypothesis in a forthcoming paper using the full sample of PHANGS targets with cloud-scale imaging by ALMA and MUSE.

\subsection{Feedback-limited lifetimes of clouds and prolonged survival of the most massive objects}

The intense feedback from the newly formed embedded stellar population in the headlight cloud is expected to lead to its eventual destruction. We appear to be observing the headlight cloud just before being destroyed by the feedback from the present generation of stars. In the simulations of Kim et al. (2018, see also Dale 2017), massive clouds with higher escape velocities can withstand destruction via photoionization and radiative pressure longer than their lower mass counterparts. For a cloud with the properties of the headlight cloud, $t_{\text {dest }} \sim 10 \mathrm{Myr}$ (Kim et al. 2018), which is greater than the age of the headlight's young stellar population. If clouds with higher masses are able to survive longer in the presence of feedback, then the overluminous state of the headlight cloud is linked not only to its star formation activity, but also to its large mass.

\subsection{Influence of galaxy dynamics on cloud growth}

If the mass of the headlight cloud is the key to its longer survival time in the presence of feedback from star formation, the next question is what mechanism(s) allowed it to grow to its extraordinary mass. The dynamical environment of the headlight offers some clues.

Massive cloud formation and incipient starbursts are often linked to extreme conditions, such as in galaxy centers, at the ends of bars, and in mergers (e.g., Kenney \& Lord 1991; Elmegreen 1994, 2011; Böker et al. 2008; Kennicutt 1998; Kormendy \& Kennicutt 2004; Beuther et al. 2017), where elevated pressures can confine highly turbulent star-forming gas (e.g., Herrera \& Boulanger 2017; Johnson et al. 2015; Leroy et al. 2018). The normal disk environment may also provide a less extreme avenue for massive cloud growth (Meidt \& Kruijssen, in prep.). As suggested earlier in Sect. 5.2, the spiral corotation radius where the headlight is positioned may furnish a favorable environment for the growth of particularly massive clouds. 
At the corotation radius of a spiral pattern, the pattern moves at the same velocity as material in the galaxy disk. An individual cloud at the intersection of a spiral arm with a corotation radius will thus reside within the spiral pattern for its entire life. Here, gas experiences no gravitational torques and shear is locally reduced, promoting the longevity (and growth) of molecular clouds situated in this zone in the absence of feedback. In Sect. 5.2 we suggested that the position of the headlight cloud at the inner corotation radius may further support the mass growth of the cloud via gas flows driven into the region by gravitational torques. Normally, an isolated corotation radius is expected to be relatively devoid of gas (Elmegreen et al. 1996), due to the inflow (outflow) of material radially inward (outward) from this point (e.g., Garcia-Burillo et al. 1994; Meidt et al. 2013; Querejeta et al. 2015). However, the inner corotation radius of NGC 628 appears to coincide with an inner dynamical resonance of the outer independently rotating spiral pattern (see bottom panel of Fig. 12). This type of scenario has been identified in simulations (Tagger et al. 1987; Rautiainen \& Salo 1999) and observations (Elmegreen et al. 2002; Meidt et al. 2009; Font et al. 2019). In this case the radial inflow of gas possible from the outer corotation radius toward the inner corotation radius provides a potential mass reservoir for the exceptional growth of the cloud (Meidt \& Kruijssen, in prep.), increasing its mass above the fiducial level set by competition between self-gravity, shear, and feedback (e.g., Reina-Campos \& Kruijssen 2017).

We can use the mass inflow rate measured in Sect. 5.2 to estimate the timescale for the growth of the headlight cloud. On the one hand, we can place a lower bound on this timescale, assuming that all of the material inflowing from the outer corotation radius is accreted onto it. The measured inflow rate in this case implies that the cloud grew to its present mass in time $t_{\text {grow }} \sim 215 \pm 100 \mathrm{Myr}$, which is roughly two orbital periods at the location of the cloud. On the other hand, considering that the inflow of gas onto the cloud is happening on multiple scales, this estimate can be viewed as an upper limit on the true growth timescale. The mechanisms by which that matter accretes onto the cloud (e.g., turbulent or gravitationally-driven accretion, Ibáñez-Mejía et al. 2017) may incorporate local material that does not originate in the outer disk, allowing condensation to proceed much more rapidly. Viscous torques would also imply a shorter growth timescale by increasing the mass inflow rate to the region.

\section{Conclusions}

We presented a detailed study of the molecular line emission and star formation activity associated with the headlight cloud. This cloud is the most massive molecular cloud in NGC 628, and it hosts an exceptionally bright H II region identified by MUSE (Kreckel et al. 2018). Our objective was to characterize the properties of this molecular cloud in the context of the internal (feedback) and external (dynamical) factors that may influence its formation and evolution.

Our main science results are as follows:

- The headlight is 2.4 times brighter than any other cloud in $\mathrm{CO}(2-1)$, and it is the only source with $\mathrm{HCO}^{+}(1-0)$, $\mathrm{HCN}(1-0)$, and $\mathrm{HNC}(1-0)$ emission detected by the ALMA $12 \mathrm{~m}$ array. The cloud is associated with the most luminous H II region in NGC 628.

- The headlight cloud is the most massive cloud in NGC 628 $\left(M_{\text {lum }}=2.0 \times 10^{7} M_{\odot}\right)$. Its virial parameter is low $\left(\alpha_{\text {vir }}=\right.$ $0.5)$. We argue that the $\mathrm{CO}$ emission is overluminous based on its high $\mathrm{CO}(2-1)$ peak temperature and its spatial coin- cidence with a bright peak in the $\mathrm{H} \alpha$ emission. This implies that its true molecular mass would be located between its virial mass of $9 \times 10^{6} M_{\odot}$ and its luminous mass $M_{\text {lum. }}$.

- According to a simple LVG analysis of the multi-line ALMA data, the typical density and temperature in the headlight cloud is $n_{\mathrm{H}_{2}} \sim 5 \times 10^{4} \mathrm{~cm}^{-3}$ and $T_{\mathrm{kin}} \sim 20 \mathrm{~K}$.

- From the presence of Wolf-Rayet features in the MUSE spectrum and modeling of the $\mathrm{H} \alpha$ emission using Starburst99, we infer the presence of a young (2-4 Myr) massive $\left(3 \times 10^{5} M_{\odot}\right)$ stellar population that is still embedded inside the headlight cloud.

- We propose that feedback from the vigorous star formation happening within the headlight is responsible for its apparent overluminous state, captured just prior to the destruction of the cloud. This is possible given the longer survival times expected for the most massive objects (e.g., Kim et al. 2018).

- We propose that the extreme headlight mass within the NGC 628 disk is related to the large-scale dynamics of the galaxy. In particular, the location of the cloud at the intersection of a spiral arm and the inner corotation radius implies not only a special location against the disruptive effects of galactic shear, but also a sustained inflow of gas favorable to enhanced mass growth. This scenario, in which clouds can grow to exceptional masses outside more prototypical extreme environments, will be discussed in more detail in a future paper by Meidt \& Kruijssen (in prep.).

The exceptional star-forming activity and physical properties of the headlight cloud call for deeper, higher angular resolution ALMA observations of tracers of the molecular gas density distribution within the cloud.

In addition to our scientific results, we developed our own CASA data reduction procedures for the ALMA total power data in order to produce the position-position-velocity cube combining the ALMA $12 \mathrm{~m}, 7 \mathrm{~m}$, and total power observations of NGC 628. These procedures, which are used in the reduction of all the total power data obtained by observational programs led by the PHANGS consortium (e.g., Leroy et al., in prep.), are described in Appendix A. As part of our PHANGS-ALMA Large Program commitments, the procedures have been transferred to the Joint ALMA Observatory so that they can be use for general purposes. Scripts implementing our procedures are also available on the PHANGS website ${ }^{5}$.

Acknowledgements. We thank the anonymous referee for a prompt and helpful report that strengthened our manuscript. This paper makes use of the following ALMA data: ADS/JAO.ALMA\#2012.1.00650.S and 2013.1.00532.S. ALMA is a partnership of ESO (representing its member states), NSF (USA) and NINS (Japan), together with NRC (Canada), NSC and ASIAA (Taiwan), and KASI (Republic of Korea), in cooperation with the Republic of Chile. The Joint ALMA Observatory is operated by ESO, AUI/NRAO and NAOJ. The National Radio Astronomy Observatory is a facility of the National Science Foundation operated under cooperative agreement by Associated Universities, Inc. We thank J. R. Goicochea for useful discussions. CNH, AH and JP acknowledge support from the Programme National "Physique et Chimie du Milieu Interstellaire" (PCMI) of CNRS/INSU with INC/INP co-funded by CEA and CNES, and from the Programme National Cosmology and Galaxies (PNCG) of CNRS/INSU with INP and IN2P3, co-funded by CEA and CNES. AU acknowledges support from the Spanish funding grants AYA2016-79006-P (MINECO/FEDER) and PGC2018-094671-B-I00 (MCIU/AEI/FEDER). The work of AKL, JS, and DU is partially supported by the National Science Foundation under Grants No. 1615105,1615109 , and 1653300. FB acknowledges funding from the European Union's Horizon 2020 research and innovation programme (grant agreement No 726384). APSH is a fellow of the International Max Planck Research School for Astronomy and Cosmic Physics at the University of Heidelberg (IMPRSHD). SCOG acknowledges support from the DFG via SFB 881 "The Milky Way System" (sub-projects B1, B2 and B8). JMDK gratefully acknowledges funding

https://sites.google.com/view/phangs/home/data 
from the European Research Council (ERC) under the European Union's Horizon 2020 research and innovation programme via the ERC Starting Grant MUSTANG (grant agreement number 714907). JMDK and MC gratefully acknowledge funding from the German Research Foundation (DFG) in the form of an Emmy Noether Research Group (grant number KR4801/1-1). SEM acknowledges funding during part of this work from the Deutsche Forschungsgemeinschaft (DFG) via grant SCHI 536/7-2 as part of the priority program SPP 1573 "ISM-SPP: Physics of the Interstellar Medium".

\section{References}

Adamo, A., Ryon, J. E., Messa, M., et al. 2017, ApJ, 841, 131 Anderson, C. N., Meier, D. S., Ott, J., et al. 2014, ApJ, 793, 37 Aniano, G., Draine, B. T., Calzetti, D., et al. 2012, ApJ, 756, 138 Barnes, J. E., \& Hernquist, L. 1996, ApJ, 471, 115

Bemis, A., \& Wilson, C. D. 2019, AJ, 157, 131

Beuther, H., Meidt, S., Schinnerer, E., Paladino, R., \& Leroy, A. 2017, A\&A, 597, A85

Bigiel, F., Leroy, A., Walter, F., et al. 2008, AJ, 136, 2846

Bigiel, F., Leroy, A. K., Jiménez-Donaire, M. J., et al. 2016, ApJ, 822, L26

Blake, G. A., Sutton, E. C., Masson, C. R., \& Phillips, T. G. 1987, ApJ, 315, 621

Blanc, G. A., Schruba, A., Evans, II, N. J., et al. 2013, ApJ, 764, 117

Blitz, L., \& Rosolowsky, E. 2006, ApJ, 650, 933

Böker, T., Falcón-Barroso, J., Schinnerer, E., Knapen, J. H., \& Ryder, S. 2008, AJ, 135, 479

Bolatto, A. D., Wolfire, M., \& Leroy, A. K. 2013, ARA\&A, 51, 207

Bosch, G., Terlevich, E., \& Terlevich, R. 2009, AJ, 137, 3437

Braine, J., Shimajiri, Y., André, P., et al. 2017, A\&A, 597, A44

Calzetti, D., Wu, S.-Y., Hong, S., et al. 2010, ApJ, 714, 1256

Calzetti, D., Lee, J. C., Sabbi, E., et al. 2015, AJ, 149, 51

Cepa, J., \& Beckman, J. E. 1990, ApJ, 349, 497

Combes, F., Debbasch, F., Friedli, D., \& Pfenniger, D. 1990, A\&A, 233, 82

Cormier, D., Bigiel, F., Jiménez-Donaire, M. J., et al. 2018, MNRAS, 475, 3909

Dale, J. E. 2017, MNRAS, 467, 1067

Druard, C., Braine, J., Schuster, K. F., et al. 2014, A\&A, 567, A118

Eckart, A., Cameron, M., Rothermel, H., et al. 1990, ApJ, 363, 451

Elmegreen, B. G. 1989, ApJ, 338, 178

Elmegreen, B. G. 1994, ApJ, 425, L73

Elmegreen, B. G. 2011, EAS Pub. Ser., 51, 45

Elmegreen, B. G., Elmegreen, D. M., Chromey, F. R., Hasselbacher, D. A., \& Bissell, B. A. 1996, AJ, 111, 2233

Elmegreen, D. M., Elmegreen, B. G., \& Eberwein, K. S. 2002, ApJ, 564, 234

Fathi, K., Beckman, J. E., Zurita, A., et al. 2007, A\&A, 466, 905

Federrath, C., \& Klessen, R. S. 2012, ApJ, 761, 156

Ferguson, A. M. N., Wyse, R. F. G., Gallagher, J. S., \& Hunter, D. A. 1998, ApJ, 506, L19

Fitzpatrick, E. L. 1999, PASP, 111, 63

Font, J., Beckman, J. E., James, P. A., \& Patsis, P. A. 2019, MNRAS, 482, 5362 Gallagher, M. J., Leroy, A. K., Bigiel, F., et al. 2018, ApJ, 858, 90 Galván-Madrid, R., Liu, H. B., Zhang, Z. Y., et al. 2013, ApJ, 779, 121

Garcia-Burillo, S., Sempere, M. J., \& Combes, F. 1994, A\&A, 287, 419

García-Burillo, S., Combes, F., Schinnerer, E., Boone, F., \& Hunt, L. K. 2005 A\&A, 441, 1011

Goicoechea, J. R., Santa-Maria, M. G., Bron, E., et al. 2019, A\&A, 622, A91

Grasha, K., Calzetti, D., Adamo, A., et al. 2015, ApJ, 815, 93

Grasha, K., Calzetti, D., Adamo, A., et al. 2017, ApJ, 840, 113

Helfer, T. T., Thornley, M. D., Regan, M. W., et al. 2003, ApJS, 145, 259

Henize, K. G. 1956, ApJS, 2, 315

Herrera, C. N., \& Boulanger, F. 2017, A\&A, 600, A139

Ho, I.-T., Medling, A. M., Groves, B., et al. 2016, Ap\&SS, 361, 280

Hughes, A., Meidt, S. E., Schinnerer, E., et al. 2013, ApJ, 779, 44

Ibáñez-Mejía, J. C., Mac Low, M.-M., Klessen, R. S., \& Baczynski, C. 2017, ApJ, 850, 62

Imanishi, M., Nakanishi, K., Tamura, Y., Oi, N., \& Kohno, K. 2007, AJ, 134, 2366

Jiménez-Donaire, M. J., Bigiel, F., Leroy, A. K., et al. 2019, ApJ, 880, 127

Johansson, L. E. B., Greve, A., Booth, R. S., et al. 1998, A\&A, 331, 857

Johnson, K. E., Leroy, A. K., Indebetouw, R., et al. 2015, ApJ, 806, 35

Kenney, J. D. P., \& Lord, S. D. 1991, ApJ, 381, 118

Kennicutt, Jr., R. C. 1998, ApJ, 498, 541

Kennicutt, R. C., \& Evans, N. J. 2012, ARA\&A, 50, 531

Kennicutt, Jr., R. C., Armus, L., Bendo, G., et al. 2003, PASP, 115, 928
Kennicutt, R. C., Calzetti, D., Aniano, G., et al. 2011, PASP, 123, 1347

Kepley, A. A., Bittle, L., Leroy, A. K., et al. 2018, ApJ, 862, 120

Kim, J.-G., Kim, W.-T., \& Ostriker, E. C. 2018, ApJ, 859, 68

Kormendy, J., \& Kennicutt, Jr., R. C. 2004, ARA\&A, 42, 603

Kreckel, K., Blanc, G. A., Schinnerer, E., et al. 2016, ApJ, 827, 103

Kreckel, K., Groves, B., Bigiel, F., et al. 2017, ApJ, 834, 174

Kreckel, K., Faesi, C., Kruijssen, J. M. D., et al. 2018, ApJ, 863, L21

Krips, M., Crocker, A. F., Bureau, M., Combes, F., \& Young, L. M. 2010 MNRAS, 407, 2261

Kruijssen, J. M. D., \& Longmore, S. N. 2014, MNRAS, 439, 3239

Kruijssen, J. M. D., Schruba, A., Hygate, A. P. S., et al. 2018, MNRAS, 479, 1866

Krumholz, M. R. 2011, AIP Conf. Ser., 1386, 9

Lang, P., Meidt, S. E., Rosolowsky, E., et al. 2019, ApJ, submitted

Leitherer, C., Schaerer, D., Goldader, J. D., et al. 1999, ApJS, 123, 3

Lelièvre, M., \& Roy, J.-R. 2000, AJ, 120, 1306

Leroy, A. K., Walter, F., Brinks, E., et al. 2008, AJ, 136, 2782

Leroy, A. K., Walter, F., Bigiel, F., et al. 2009, AJ, 137, 4670

Leroy, A. K., Walter, F., Sandstrom, K., et al. 2013, AJ, 146, 19

Leroy, A. K., Hughes, A., Schruba, A., et al. 2016, ApJ, 831, 16

Leroy, A. K., Bolatto, A. D., Ostriker, E. C., et al. 2018, ApJ, 869, 126

Leroy, A. K., Sandstrom, K. M., Lang, D., et al. 2019, ApJS, 244, 24

Liu, J. 2005, M74: X-rays Signal Presence of Elusive Intermediate-Mass Black Hole (Chandra Press Release CXC:05-04)

Meidt, S. E., Rand, R. J., \& Merrifield, M. R. 2009, ApJ, 702, 277

Meidt, S. E., Schinnerer, E., García-Burillo, S., et al. 2013, ApJ, 779, 45

Meidt, S. E., Schinnerer, E., van de Ven, G., et al. 2014, ApJ, 788, 144

Mulcahy, D. D., Beck, R., \& Heald, G. H. 2017, A\&A, 600, A6

Nishimura, Y., Shimonishi, T., Watanabe, Y., et al. 2016, ApJ, 829, 94

Ostriker, E. C., McKee, C. F., \& Leroy, A. K. 2010, ApJ, 721, 975

Owen, R. A., \& Warwick, R. S. 2009, MNRAS, 394, 1741

Pellegrini, E. W., Baldwin, J. A., Brogan, C. L., et al. 2007, ApJ, 658, 1119

Pety, J., Schinnerer, E., Leroy, A. K., et al. 2013, ApJ, 779, 43

Pety, J., Guzmán, V. V., Orkisz, J. H., et al. 2017, A\&A, 599, A98

Pineda, J. L., Mizuno, N., Röllig, M., et al. 2012, A\&A, 544, A84

Querejeta, M., Meidt, S. E., Schinnerer, E., et al. 2015, ApJS, 219, 5

Querejeta, M., Meidt, S. E., Schinnerer, E., et al. 2016, A\&A, 588, A33

Rahner, D., Pellegrini, E. W., Glover, S. C. O., \& Klessen, R. S. 2017, MNRAS, 470, 4453

Rautiainen, P., \& Salo, H. 1999, A\&A, 348, 737

Rebolledo, D., Wong, T., Xue, R., et al. 2015, ApJ, 808, 99

Reina-Campos, M., \& Kruijssen, J. M. D. 2017, MNRAS, 469, 1282

Rogers, H., \& Pittard, J. M. 2013, MNRAS, 431, 1337

Rosolowsky, E., \& Leroy, A. 2006, PASP, 118, 590

Rousseau-Nepton, L., Robert, C., Martin, R. P., Drissen, L., \& Martin, T. 2018, MNRAS, 477, 4152

Ryon, J. E., Gallagher, J. S., Smith, L. J., et al. 2017, ApJ, 841, 92

Saintonge, A., Catinella, B., Tacconi, L. J., et al. 2017, ApJS, 233, 22

Sakamoto, S., Hayashi, M., Hasegawa, T., Handa, T., \& Oka, T. 1994, ApJ, 425, 641

Sakamoto, S., Hasegawa, T., Handa, T., Hayashi, M., \& Oka, T. 1997, ApJ, 486, 276

Sánchez, S. F., Rosales-Ortega, F. F., Kennicutt, R. C., et al. 2011, MNRAS, 410, 313

Schinnerer, E., Meidt, S. E., Pety, J., et al. 2013, ApJ, 779, 42

Schruba, A., Leroy, A. K., Walter, F., Sandstrom, K., \& Rosolowsky, E. 2010, ApJ, 722, 1699

Seale, J. P., Looney, L. W., Wong, T., et al. 2012, ApJ, 751, 42

Sheth, K., Regan, M., Hinz, J. L., et al. 2010, PASP, 122, 1397

Solomon, P. M., Rivolo, A. R., Barrett, J., \& Yahil, A. 1987, ApJ, 319, 730

Sun, J., Leroy, A. K., Schruba, A., et al. 2018, ApJ, 860, 172

Tagger, M., Sygnet, J. F., Athanassoula, E., \& Pellat, R. 1987, ApJ, 318, L43

Usero, A., Leroy, A. K., Walter, F., et al. 2015, AJ, 150, 115

Utomo, D., Sun, J., Leroy, A. K., et al. 2018, ApJ, 861, L18

van Dishoeck, E. F., \& Black, J. H. 1988, ApJ, 334, 771

van Marle, A. J., Meliani, Z., \& Marcowith, A. 2015, A\&A, 584, A49

Wakker, B. P., \& Adler, D. S. 1995, AJ, 109, 134

Walborn, N. R. 1991, IAU Symp., 148, 145

Walter, F., Brinks, E., de Blok, W. J. G., et al. 2008, AJ, 136, 2563

Watanabe, Y., Nishimura, Y., Harada, N., et al. 2017, ApJ, 845, 116

Wong, T., \& Blitz, L. 2002, ApJ, 569, 157

Wong, T., Blitz, L., \& Bosma, A. 2004, ApJ, 605, 183

Wong, T., Hughes, A., Ott, J., et al. 2011, ApJS, 197, 16

Yoda, T., Handa, T., Kohno, K., et al. 2010, PASJ, 62, 1277 


\section{Appendix A: Total power data reduction}

We have developed a set of customized scripts to carry out ALMA single-dish (total power) data reduction ${ }^{6}$. These scripts play a crucial role in the PHANGS-ALMA reduction pipeline as the short spacings often contain a significant fraction of the flux of nearby galaxies. They are based on the ALMA calibration scripts, with modifications informed by our experience carrying out and supporting single-dish data reduction using the GILDAS software for IRAM $30 \mathrm{~m}$ data. The main aim of the scripts is to allow the user to define a fixed velocity window for the baseline subtraction while also producing a suite of diagnostic plots that allow assessment of imaging quality. This appendix describes the structure and usage of these scripts.

The scripts were provided to the ALMA Regional Centers and the Joint ALMA Observatory, where they have helped inform development of the Observatory data reduction scheme. They are available on GitHub ${ }^{7}$.

They were developed to be used in CASA version 4.7.2, which uses the ATNF Spectral Analysis Package (ASAP) extension to deal with total power gridding and baselining. Since then, newer versions of CASA have included dedicated packages that perform these tasks. We have not ported our scripts yet.

The script distribution contains three files:

analysis_scripts: ALMA-provided folder that contains useful tools for TP ALMA data reduction. The scripts in this folder should not be modified.

ALMA-TP-tools . py: Script which contains our generic procedures for total power data reduction and imaging. This script should not be modified.

galaxy-specific-scripts: Folder containing the individual galaxy-specific scripts, GalaxyName-input.py, which are a wrapper for the two previous scripts. Here the user specifies various galaxy- and project-specific imaging parameters, including phase center position, source systemic velocity, rest frequency, channel width, final cube velocity width, and the definition of baseline subtraction windows (see additional details in the end-to-end reduction description below).

The distributed code must be saved in a folder named scripts_TP folder. The galaxy-specific GalaxyName-input.py CASA script should be executed in this folder. Two additional folders will be created at the same level as the scripts_TP folder, data and tmp. The first will contain the output cube fits files. The second is a temporary folder where the total power data reduction happens and intermediate files are stored. Once the data reduction is done, this folder can be deleted. Total power raw data stored in the original ALMA directory structure are first copied in the tmp folder. Only the needed files are copied. Data reduction products are created in the calibration folder. Specifically, two additional subfolders are created, plots and obs_lists. The plots folder contains all plots created by the data reduction scripts, for example the $T_{\text {sys }}$ and the baseline correction plots. The quality of the data can be judged checking these plots. The obs_lists folder contains the observation lists for the data.

The main script, ALMA-TP-tools.py, contains the procedures and tasks for the data reduction. We now describe the main steps in order of their execution during the processing.

\footnotetext{
6 Cinthya N. Herrera wrote the scripts guided by Jérôme Pety. Christopher Faesi and Antonio Usero extensively used these scripts and proposed important upgrades.

https://sites.google.com/view/phangs/home/data
}

Import data to MS and split by antenna. The raw ALMA data are stored in the Archival Science Data Model (ASDM) format. The data are converted to measurement sets (MSs), a CASA table format, using the task importasdm. Initial flagging is applied with the task flagcmd. These are a priori flags, which are already encoded in and read from the MSs themselves. These flags could include issues, for example when the mount is off source, the calibration device is in an incorrect position, or the power levels are not optimized. We also flag $7 \mathrm{~m}$ antennas that may have been used for total power observations. Using the task sdsave, each execution block dataset in the MS format is split by antenna and converted to the ASAP format.

Generate $T_{\text {sys }}$ calibration tables and apply additional flags. For each ASAP file, a system temperature $\left(T_{\text {sys }}\right)$ calibration table is generated using the task gencal, which is needed to apply the calibration scale to the observations. One $T_{\text {sys }}$ solution is created per spectral window. In this step additional flagging to the data can also be applied by creating a script containing custom flag commands. This script should be placed in the galaxy-specific-scripts/flags-folder folder.

Subtract the atmospheric total power and convert to Kelvin. The $T_{\text {sys }}$ calibration solution is applied to the data with the task sdcal2. At the same time, this task subtracts the contribution of the atmosphere to the total power (subtraction of the OFF measurement) and it corrects for the chromatic response of the atmosphere and the receivers by dividing by the OFF measurement. The output will thus be expressed in units of antenna temperature as

$$
T_{\mathrm{a}}^{\star}=T_{\mathrm{sys}} \times \frac{(\mathrm{ON}-\mathrm{OFF})}{\mathrm{OFF}}
$$

Extract the frequency window that contains the emission line. From this step onward, we choose to continue the data processing only for the spectral window that contains the emission line of interest plus a baseline margin on each side of the line. The user must define in the script GalaxyName-input.py both the rest frequency of the emission line and the velocity range of the cube that will be extracted from the data. The systemic velocity of the source is read from the MS. As the ALMA data are originally observed in the TOPO frame, the spectral axis is converted to the LSRK velocity frame at this stage to simplify the extraction. This uses functionality inside the analysis_scripts folder. The extraction of the cube is done with the task sdsave.

Baseline the spectra. We fit a polynomial though the spectrum parts that belong to the velocity ranges on both side of the lines. The velocity range to be ignored in this baseline fitting is defined in the GalaxyName-input . py script. More than one velocity window can be defined. The order of the baseline fit is one (i.e., a linear fit) by default, but this can also be changed in the GalaxyName-input . py script. Baseline fitting and subtraction call the sdbaseline task.

Convert to Jy/Beam and concatenate data from all antennas. After all the above steps for data calibration are done, the ASAP files for all execution blocks and antenna are converted back to MS format using the task sdsave. For each execution block and individual antenna, the data are converted from kelvin to jansky using the factor measured and delivered by ALMA based on independent observations of point sources of known flux. This makes a higher order correction to the flux scale. All the data are then concatenated using the task concat. 

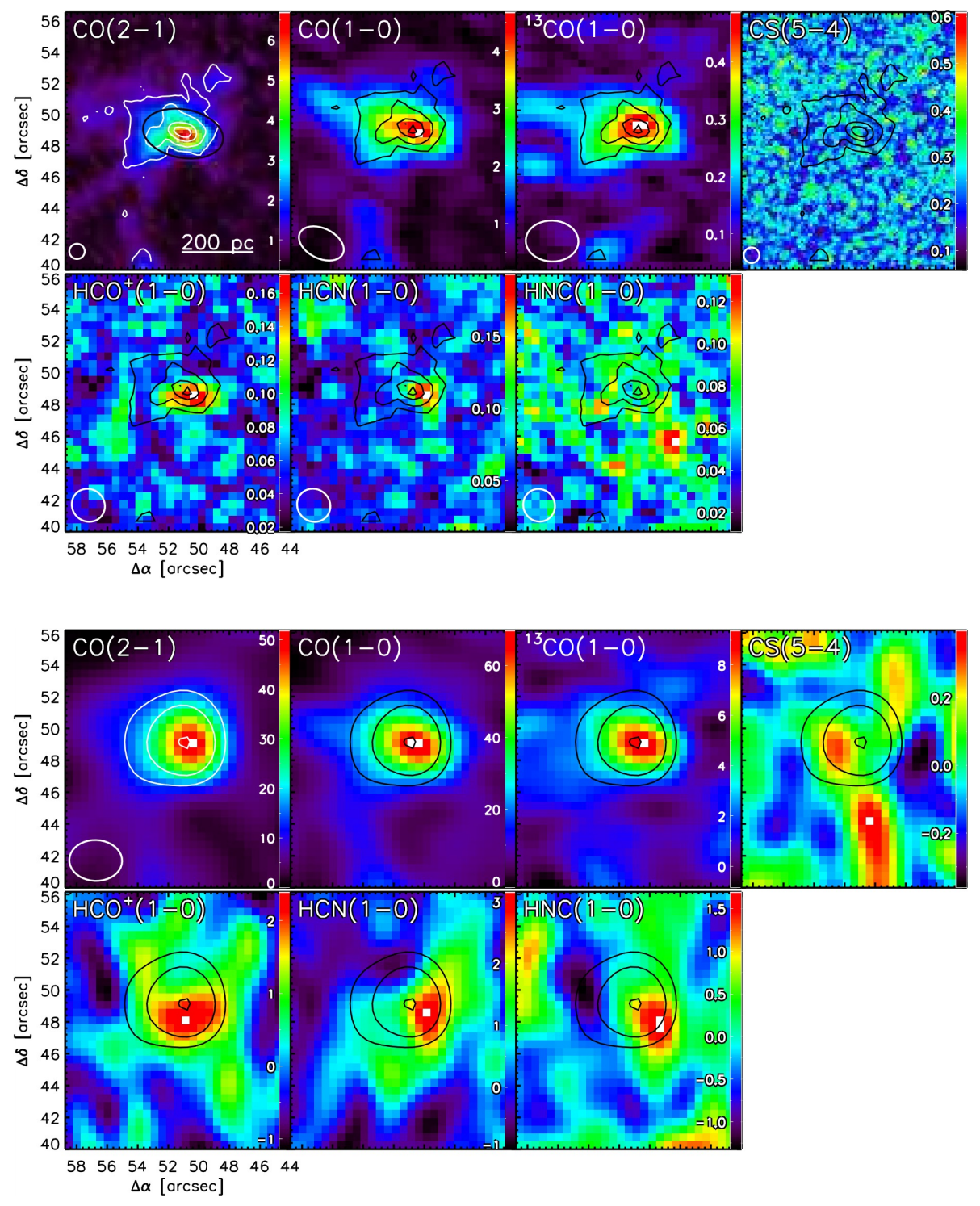

Fig. B.1. Peak temperature for the different gas tracers observed with ALMA. The size of the synthesized beam is shown in the bottom left corner of each panel. The color bars are in units of kelvin. The white contours in the first panel (black contours in the other panels) correspond to the $20 \%, 40 \%, 70 \%$, and $90 \%$ of the $\mathrm{CO}(2-$ 1) peak temperature. Top left panel: headlight cloud as identified by CPROPS (black ellipse). Offset positions are from the galactic center.

Fig. B.2. Integrated emission line for the different gas tracers observed with ALMA, convolved to the spatial resolution of ${ }^{13} \mathrm{CO}(1-0)$. The size of the synthesized beam is shown in the bottom left corner of the first panel. The emission for all tracers is integrated between 650 and $685 \mathrm{~km} \mathrm{~s}^{-1}$; the color bars are in units of $\mathrm{K} \mathrm{km} \mathrm{s}^{-1}$. The white contours in the first panel (black contours in the other panels) correspond to $15 \sigma, 25 \sigma, 50 \sigma$, and $100 \sigma$, with $\sigma=1.01 \mathrm{~K} \mathrm{~km} \mathrm{~s}^{-1}$ of the $\mathrm{CO}(2-1)$ line. Offset positions are from the galactic center.

Grid the spectra onto a pixelized cube. All the execution blocks together are imaged together. The user can define the imaging parameters such as the projection center, the velocity frame of reference, the total velocity width, and the channel width in the GalaxyName-input . py script. The gridding is done using the sdimaging task, in the LSRK velocity reference frame using a spheroidal function.

Export the position-position-velocity to a fits file. The task exportfits is used to export the signal and weight cubes to fit files.

In a few cases an ozone telluric line was not satisfactorily subtracted during the ON-OFF processing because the atmosphere contribution to the total power varied too quickly. We used an advanced baselining functionality in the GILDAS/CLASS software to remove this artifact and to obtain flat baselines. A deeper analysis that will be presented elsewhere (Usero et al., in prep.) showed that a change of observing mode is sometimes required to get rid of or at least decrease the contribution from some ozone lines.

\section{Appendix B: Headlight spatial distributions of multiple tracers}

Figure B.1 shows the spatial distribution at full angular resolution of the peak brightness temperature around the headlight for the observed molecular lines: $\mathrm{CO}(2-1), \mathrm{CO}(1-0),{ }^{13} \mathrm{CO}(1-0)$, $\mathrm{CS}(5-4), \mathrm{HCO}^{+}(1-0), \mathrm{HCN}(1-0)$, and $\mathrm{HNC}(1-0)$. Figure B.2 shows the spatial distribution of the emission integrated over the line profiles for the same tracers after convolution to the coarsest angular resolution obtained (i.e., the ${ }^{13} \mathrm{CO}(1-0)$ ). These figures show that $\mathrm{HCO}^{+}(1-0), \mathrm{HCN}(1-0)$, and $\mathrm{HNC}(1-0)$ are clearly detected in contrast with $\mathrm{CS}(5-4)$. The high-density tracers are slightly offset compared to the CO isotopologue lines. The relatively low signal-to-noise ratio of the $\mathrm{HCO}^{+}, \mathrm{HCN}$, and $\mathrm{HNC}$ lines makes the origin of the offsets uncertain. 